\title{
MODELS OF CHEMICAL REACTIONS WITH PARTICIPATION OF POLYMERS
}

\section{G. OSHANIN ${ }^{\mathrm{a}, *}$, M. MOREAU ${ }^{\mathrm{a}}$ and S. BURLATSKY $\mathrm{M}^{\mathrm{b}, \mathrm{c}}$}

${ }^{a}$ Laboratoire de Physique Theorique des Liquides, Université P. et M. Curie, 4, place Jussieu, 75252 Paris, France

${ }^{\mathrm{b}}$ Institute of Chemical Physics, Kosygin st.4, Moscow V-334, Russian Federation 'Department of Chemistry, Massachusetts Institute of Technology, Cambridge, MA 02139, USA

\section{ABSTRACT}

In this paper we review some recent results on the kinetics of bimolecular chemical reactions, such as binary, recombination and trapping reactions, with participation of polymer chains. We consider both mean-field and many-particle approaches to the description of reaction kinetics in such systems, present explicit dependences describing evolution of particle concentrations and discuss the origins of anomalous kinetic behavior.

\section{INTRODUCTION}

Chemical reactions involving reactants which execute random motion represent an example of systems with dynamical essentially many-particle behavior. An attempt to devise an adequate analytical description of the reaction kinetics is limited by the usual difficulties of genuine many-particle problems - infinite hierarchies of coupled nonlinear equations, diverging perturbation-theory series or substantial importance of irreducible diagrams. Consequently, certain approximations or complicated statistical physics methods must be invoked in order to develop a tractable and reliable theory of transport-controlled chemical reactions. A great deal of effort has been devoted to the elaboration of such analytical approaches. Several excellent books [1-3], reviews [4-12] and a series of original papers [13-28] provide details of the numerous advances this field has experienced during the last eight decades.

* Present address: Theoretical Polymer Physics, University of Freiberg, Rheinstrasse 12, D-79104 Freiberg, Germany. 
Chemical reactions with participation of polymers is one of the most practically important areas among the general field of transport-controlled processes. Polymerization reactions, cyclization processes, polymersupported catalysts, energy transfer in polymer matrix are a few typical examples. Reaction kinetics in polymer systems have been also widely studied within recent decades [29-31]. A considerable progress has been gained in the understanding of chemical reactions taking place in polymer systems. This is due primarily to classical works [32-35] on the kinetics of cyclization processes, and works by de Gennes [31], who established the relation between anomalous kinetics of bimolecular chemical reactions in polymer systems and transport properties of polymers.

In this paper we summarize some recent results on the kinetics of bimolecular transport-controlled reactions, described by the reaction schemes

(II) $A+B \rightarrow P$

(III) $A+C \rightarrow C+P$

taking place in polymer systems. These include bimolecular reactions between active groups attached to flexible polymer chains (Fig. 1), polymerization and trapping reactions in polymer systems. This paper is by no means a complete review of results in the field of chemical reactions in polymer systems and it would require far too much space to make it so. For instance, we do not consider here the analysis of cyclization processes kinetics and address the reader to the original works on this subject $[29,30,32-35]$.

The present paper is organized as follows: in the first section we state the results of traditional approaches - the formal kinetic scheme and the Smoluchowski approach to the kinetics of reactions I-III taking place in the simplest chemical systems, i.e. systems in which spherical particles presented at small initial concentrations execute conventional diffusive motion and react when they "meet each other". Although there exists a vast literature on this subject we have found it necessary to present their basic statements and results here, since the traditional approaches, especially the Smoluchowski approach, will be very useful for the explanation of different questions related to chemical kinetics in polymer systems. In Section 2 we briefly discuss the results of many-particle 


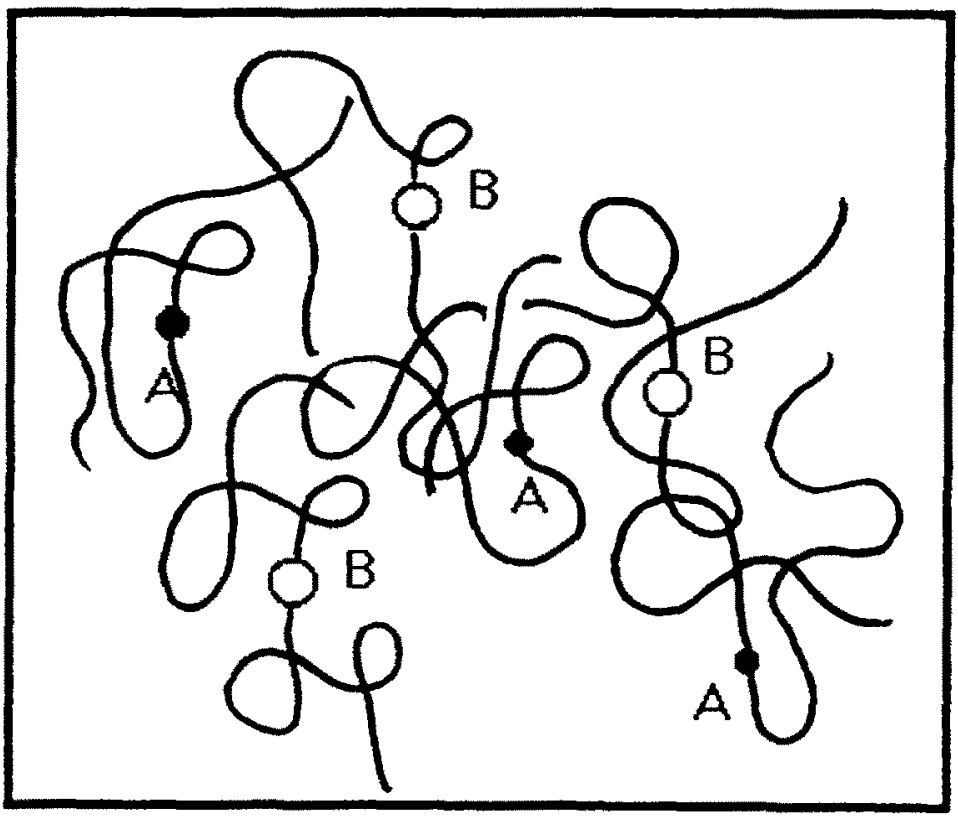

Fig. 1. Chemical system with recombination reaction, $A+B \rightarrow P$, involving active particles $A$ and $B$ attached to flexible polymer chains in polymer solution.

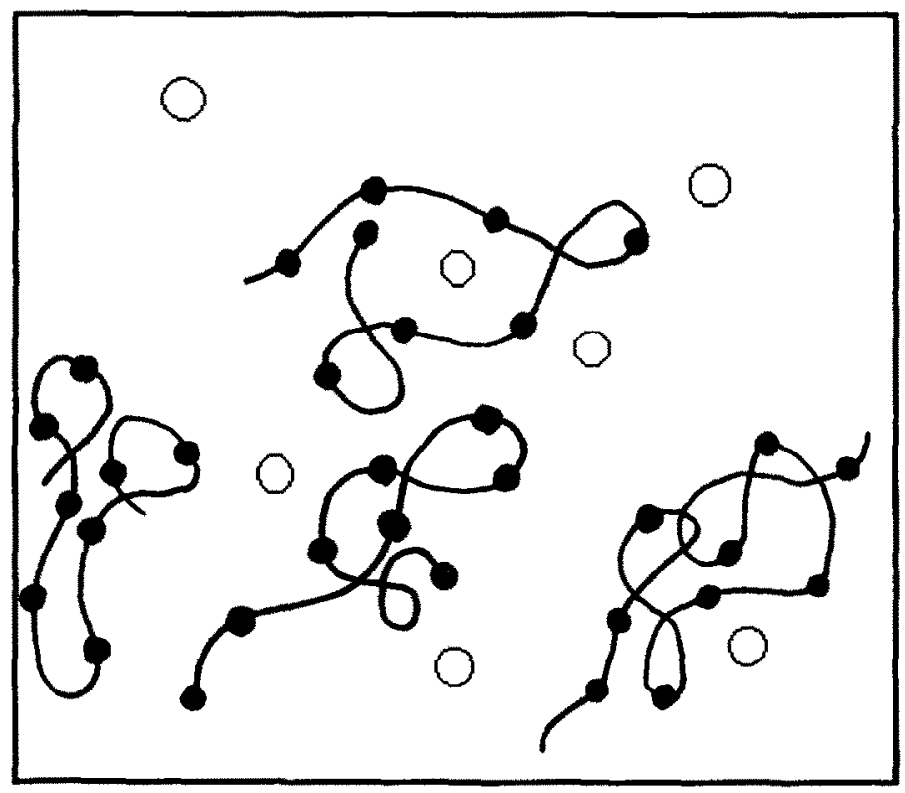

Fig. 2. Trapping reaction in systems with polymerized traps. Open circles denote diffusive particles $\mathrm{A}$. Solid circles are traps $\mathrm{C}$ attached to immobile polymer chains. 
approaches to chemical reactions kinetics. In Section 3 we discuss the extensions of the Smoluchowski approach to the kinetics of reactions I-III taking place in polymer systems. We will consider reactions between active groups attached to warmlike polymers in concentrated or diluted polymer solutions (Fig. 1), kinetics of polymerization reactions in systems with transport limitations and kinetics of trapping-like reactions in systems with polymerized traps (Fig. 2). Finally, in Section 4 we present results of several many-particle considerations of reaction kinetics in polymer solutions.

\section{CONVENTIONAL APPROACHES TO CHEMICAL KINETICS IN THE "SIMPLEST" CHEMICAL SYSTEMS}

To establish notation, we begin with the definitions of the "simplest" chemical systems and standard treatments of the reaction kinetics in such systems. On base of the reaction scheme III we will discuss the results of the formal kinetic scheme and of the Smoluchowski approach. We will also mention the predictions of these approaches for the kinetics of binary (I) and recombination (II) reactions. More involved analysis of this subject and several important extensions to more complicated reaction schemes can be found in [1,4-6,13-18].

Consider a d-dimensional reaction bath of volume $V(V \rightarrow \infty$, so that boundary effects can be neglected) with reactive species of two types $A$ and $C$. We assume that $A$ and $C$ are spherical and noninteracting. Particles diffuse freely in the reaction bath with well-defined diffusion constants $D_{A}$ and $D_{C}$ (solute is homogeneous everywhere and does not impose any restrictions on the diffusion). Thus, in the absence of reaction between $A$ and $C$ their dynamics are described by a diffusion equation.

Whenever an $A$ and a $C$ come in contact at separation $R$ (reaction radius) particle $A$ is annihilated and the reaction product $P$ is formed with some finite probability determining the reaction constant $K_{c h e m}$. The reaction constant $K_{\text {chem }}$ describes the intrinsic properties of the elementary reaction act.

The evolution of local concentration of particles $A$ (concentration at point $r$ at time $t$ ) due to reaction and diffusion is governed by the following equation

$\frac{\partial C_{A}(r, t)}{\partial t}=-K_{c h e m} \int d r_{1} C_{A}(r, t) C_{C}\left(\left|r-r_{1}\right|=R, t\right)+D_{A} \Delta_{r} C_{A}(r, t)$ 
where $\Delta_{r}$ denotes d-dimensional diffusion operator (Laplacian). The first term in the right-hand-side of Eqn. (1a) defines the loss of particles $A$ due to trapping of $A$ by $C$. The integration over $r_{1}$ accounts for the fact that reaction takes place when particles approach each other at fixed separation distance $R$. The second term in the right-hand-side of Eqn. (1) describes the diffusive smoothing of spatial inhomogeneities in particles concentrations. These inhomogeneities can be macroscopic and microscopic. The macroscopic inhomogeneities can be introduced into the reactive system by the method of preparation of the initial mixture of reactants, e.g. initially particles can be generated in cascades or introduced by external source on the surface of the reaction bath. Microscopic inhomogeneities appear due to thermal fluctuations in particles concentrations and in the reaction course.

Concentration of traps $C$ is not affected by the reaction act and changes only due to diffusion,

$$
\frac{\partial C_{C}(r, t)}{\partial t}=D_{C} \Delta_{r} C_{C}(r, t)
$$

Similar equations govern the evolution of local concentrations in the course of binary irreversible reaction (I),

$\frac{\partial C_{A}(r, t)}{\partial t}=-K_{\text {chem }} \int d r_{1} C_{A}(r, t) C_{A}\left(\left|r-r_{1}\right|=R, t\right)+D_{A} \Delta_{r} C_{A}(r, t)$

and irreversible recombination reaction (II)

$$
\begin{aligned}
& \frac{\partial C_{A}(r, t)}{\partial t}=-K_{c h e m} \int d r_{1} C_{A}(r, t) C_{B}\left(\left|r-r_{1}\right|=R, t\right)+D_{A} \Delta_{r} C_{A}(r, t) \\
& \frac{\partial C_{B}(r, t)}{\partial t}=-K_{\text {chem }} \int d r_{1} C_{A}\left(\left|r-r_{1}\right|=R, t\right) C_{B}(r, t)+D_{B} \Delta_{r} C_{B}(r, t)
\end{aligned}
$$

The quantities which are observed experimentally are the mean concentrations of particles,

$C_{A, B}(t)=\left\langle C_{A, B}(r, t)\right\rangle$

where brackets stand for volume averages. However, evaluation of analytical dependences which describe the temporal evolution of observables 
turns out to be a complicated mathematical problem. If one writes down corresponding equations for the evolution of mean concentration, one finds that behavior of $C_{A, B}(t)$ is coupled to the behavior of pairwise correlation functions. For example, for the recombination reaction II averaging of Eqns. (3) yields,

$\frac{d C_{A}(t)}{d t}=\frac{d C_{B}(t)}{d t}=-K_{\text {chem }}\left[C_{A}(t) C_{B}(t)+P_{A B}(|\lambda|=R, t)\right]$

where

$P(\lambda, t)=\left\langle\delta C_{A}(r, t) \delta C_{B}(r+\lambda, t)\right\rangle$

is the pairwise $\mathrm{A}-\mathrm{B}$ correlation function, $\lambda$ is correlation parameter, and $\delta C_{A, B}(r, t)$ denote local deviations of particles concentrations from the mean values,

$\delta C_{A, B}(r, t)=C_{A, B}(r, t)-C_{A, B}(t)$

By definition, $\left\langle\delta C_{A, B, C}(r, t)\right\rangle=0$, but the average product of local deviations, $P_{A B}(\lambda, t)$, is not zero. Equations describing the evolution of the pairwise correlations are coupled to the third-order correlation functions and so on. Therefore, one is faced with the infinite hierarchy of coupled differential equations. Moreover, even in the absence of macroscopic inhomogeneities initial concentrations of particles are perturbed by thermal fluctuations and one thus has random initial conditions to this hierarchy. In the remaining part of this section and in Section 2 we will consider different approaches to the solution of Eqns. (1-4) and discuss the behavior of the observables.

\subsection{Formal kinetic scheme}

Let us begin with the simplest approach to Eqns. (1-4). Suppose that the reaction constant $K_{\text {chem }}$ is small enough, so that the elementary reaction act - reaction between $A$ and $C$ being at the separation distance $R$ can be thought of as an essentially slower process as compared with the transport of particles to each other. Particles $A$ and $C$ meet each other many times before $A$ is annihilated and the reaction product is formed. Under such conditions one can assume that the limiting stage of reaction is the elementary reaction act and, besides, that diffusion perfectly mixes 
the species in the reaction bath. Therefore, one can neglect (not safely, as we will see below) fluctuations $\delta C$ in spatial distributions of species, i.e. stipulate that everywhere in the reaction bath particles concentrations are equal to their mean values $C_{A}(t)$ and $C_{C}$ which depend on time only. Equations (1) and (2) then reduce to the "law of mass action"

$\frac{d C_{A}(t)}{d t}=-K_{\text {chem }} C_{C} C_{A}(t)$

with the intrinsic reaction constant $K_{\text {chem }}$ calculated by means of standard methods $[1,2,7,19,25,26]$. The solution of (5) (which is essentially $\mathrm{d}$-independent since the diffusion operator is skipped) predicts a simple exponential decay of $A$ particles,

$\log C_{A}(t) \propto-K_{\text {chem }} C_{C} t$

For the reaction schemes I and II such an approach results in the following decay patterns. For the binary reaction one obtains

$C_{A}(t) \propto \frac{1}{K_{\text {chem }} t}$

for times $t$ sufficiently large. Kinetics of the recombination reaction are sensitive to the relation between initial concentrations of $A$ and $B$ particles. In case of equal initial concentrations (further on we will term this case as $\mathrm{IIa}$ ) the kinetics are governed by Eqn. (7). In the case of unequal initial concentrations (IIb) the minority component decays via the dependence in Eqn. (6) while for the majority component Eqn. (6) defines the long-time approach to the equilibrium concentration.

\subsection{Smoluchowski approach}

The formal kinetic scheme rests on the implicit assumption that the reaction process consists of two independent stages - transport of two reagents to each other and the elementary reaction act. Besides, it is assumed that these stages are comparable in the sense that diffusion of species or the transport stage can only modify the reaction constant but can not change the time dependence of particle concentrations. A natural question is to understand what are the actual limitations of random transport of particles and how they might control the kinetics of chemical 
reactions. This question has been first addressed by Smoluchowski [13] in his classic study of the kinetics of coagulation in colloidal solutions. He proposed to account for the random (diffusive) transport of particles in the following manner.

(a) Statements and results of Smoluchowski approach

Let us suppose that one can neglect many-trap effects, e.g. screening of a given trap by traps placed in its vicinity. Then the initial problem can be reduced to the model with a single immobile trap of radius $R$ placed in the "sea" of infinitesimal diffusing particles $A$. At $t=0$ particles are scattered uniformly throughout space. Particles $A$ diffuse, with diffusion constant $D_{+}=D_{C}+D_{A}$, and disappear as soon as they encounter the surface of the trap. The probability that particle $A$ survives until time $t$ is then defined by the diffusion equation

$$
\frac{\partial P(r, t)}{\partial t}=D_{+} \Delta_{r} P(r, t)
$$

subject to the following boundary conditions: $P(r, t=0)=P(r \rightarrow \infty, t)=1$, and an absorbing boundary condition at separation distance $R$,

$P(r=R, t)=0$

which mimics the reaction between species*. From the solution of Eqns. (8) one can compute the number of reaction events very directly. The apparent rate constant, or the Smoluchowski constant (SC), equals the diffusive current

$K_{S m o l}(t)=\int_{S(R)} d S J(R, t) ; J(R, t)=-\left.D_{+} \frac{\partial P(r, t)}{\partial r}\right|_{r=R}$

integrated over the trap's surface $S(R)$. Evolution of $C_{A}(t)$ is then described by equation similar to the law of mass action but with the Smoluchowski rate constant

$$
\frac{d C_{A}(t)}{d t}=-K_{S m o l}(t) C_{C} C_{A}(t)
$$

* Within the assumption that $K_{\text {chem }}=\infty$. 
Consider next the behavior of SC in systems of different spatial dimensions. In three-dimensional (3d) systems the survival probability $P(r, t)$ reaches after some time a steady state profile and, consequently, the diffusive current and $K_{S m o l}(t)$ approach constant values as $t$ tends to infinity. In $3 \mathrm{~d}$ the complete time dependence of SC can be computed

$K_{S m o l}(t)=4 \pi R D_{+}\left[1+\frac{R}{\sqrt{\pi D_{+} t}}\right]$

where the first term defines the steady state value and the second time-dependent term represents the transient regime associated with the formation of the depletion zone around the trap. The transient regime takes place until times $t$ less than $R^{2} / D_{+}$. The decay patterns in $3 \mathrm{~d}$ systems can be easily found. For (III) and (IIb) one obtains an exponential dependence of the form

$\log C_{A}(t) \propto-4 \pi R D_{+} C t\left[1+R(4 \pi D t)^{-1 / 2}\right]$

while the kinetics of (I) and (IIa) are governed by an algebraic dependence

$C_{A}(t) \propto \frac{1}{4 \pi R D_{+} t\left[1+R(4 \pi D t)^{-1 / 2}\right]}$

Therefore, in 3d systems the Smoluchowski approach predicts essentially the same time dependences of mean particles concentrations as the formal kinetic scheme, except the transient regime connected with the formation of the steady state rate constant in Eqn. (10). It means that in $3 \mathrm{~d}$ (in terms of the Smoluchowski approach) the reaction process can be represented in the form of two independent reaction stages and one can distinguish between kinetic-controlled reactions and diffusion-controlled reactions.

However, there is a notable distinction between the predictions of the formal kinetic scheme and the Smoluchowski approach in case of low dimensional $(d \leq 2)$ reaction systems. Let us note that the steady state form in Eqn. (10) is specifically 3d solution of the diffusion equation with Smoluchowski boundary conditions. If we consider the same problem in $1 \mathrm{~d}$ (or $2 \mathrm{~d}$ ) we find completely different behavior. There is no steady state profile around the reaction sphere but a hole, or depletion zone, which grows with time. This results in the anomalous behaviour of SC - it does not reach a steady state value as $t \rightarrow \infty$ but decreases with time as 
$K_{S m o l}(t) \propto\left(D_{+} / t\right)^{1 / 2}, d=1$

$K_{S m o l}(t) \propto D_{+} / \log \left(D_{+} t / R^{2}\right), D_{+} t>>R^{2}, d=2$

The behavior of the observables will thus depend essentially on the dimensionality of the reaction bath. In low dimensional systems one will obtain for I and IIa anomalous dependences

$C_{A}(t) \propto \sqrt{1 / D_{+} t}, d=1$

$C_{A}(t) \propto \log \left(D_{+} t / R^{2}\right) / D_{+} t, d=2$

and for III and IIb stretched-exponential decays of the form

$\log C_{A}(t) \propto-\sqrt{D_{+} t}, d=1$

$\log C_{A}(t) \propto-D_{+} t / \log \left(D_{+} t / R^{2}\right), d=2$

Therefore, assuming that $K_{c h e m}=\infty$ and accounting for the random diffusive motion of reactive species we are led to a very different behavior as compared to the formal kinetic scheme predictions. A striking distinction is that the form of decay functions does depend on the dimensionality of reactive systems. This observation leads to very important consequences. Let us consider the behavior of the rate constant in Eqn. (9) in systems with finite intrinsic rate constant $K_{\text {chem. }}$. It was proposed [15] to account for the finite intrinsic rate by imposing another type of boundary condition to Eqn. (8) - a radiation boundary condition instead of the absorbing boundary condition in Eqn. (8b). Within such an approach one finds [15] from Eqns, $(8,9)$ the "apparent" rate constant which accounts for both the diffusive transport of particles and the intrinsic reaction rate. This "apparent" rate constant was found [15] to obey the following "inverse resistivity" law

$\frac{1}{K_{\text {app }}}=\frac{1}{K_{\text {chem }}}+\frac{1}{K_{\text {Smol }}}$

where $K_{\text {Smol }}$ is defined by Eqns. $(10,13)$. It follows from Eqn. (16) that in $3 \mathrm{~d}$, depending on the relation between constants, reaction can be either diffusion-controlled or kinetic-controlled. However, in low dimensions, 
the long-time kinetics will be always diffusion-controlled since $K_{S m o l}$ tends to zero as $t \rightarrow \infty$, i.e. in low dimensional systems random transport of particles to each other has essentially higher "resistivity" than the rate of elementary reaction act.

The final point which has to be mentioned is the question of what is the marginal dimension of the reactive system below which one can expect "anomalous" behavior of the Smoluchowski constant. In the considered above case of the "simplest" chemical systems with diffusive reactants this marginal dimension $d_{m}$ is equal to 2 . However, as we shall show below, $d_{m}=2$ is only a particular value which is associated with the particular type of random motion -conventional diffusion. In systems with reactions involving polymers $d_{m}$ will be greater than 2 and, in general, will depend on time.

\section{(b) Geometric interpretation of the Smoluchowski constant}

Next we would like to mention a geometric interpretation of the Smoluchowski constant, which is less familiar than the usual definition in Eqns. (8,9).

Let us consider kinetics of trapping reactions on a d-dimensional lattice in which each lattice site can be a trap with probability $C_{C}$. Let us suppose next that the random walk performed by particles $A$ takes place in discrete time. Then the mean concentration of particles $A$ is governed by $[5,22,36,37]$

$$
C_{A}(n)=C_{A}(0)\left\langle\left(1-C_{C}\right)^{S(n)}\right\rangle
$$

where the function $S(n)$ is the number of distinct sites visited by $A$ particle during time $n$; brackets denote the average both over all trap configurations and realizations of random walk. Equation (17) is exact but not very useful since the average cannot be taken explicitly and one has to resort to approximate methods and examine separately the behavior of $C_{A}(n)$ at small and large times $n$. The large- $n$ behavior of $C_{A}(n)$ is dominated by many-particle effects and will be discussed in the next section. Here we consider a simple bound on $C_{A}(n)$ - the so-called Rosenstock approximation [38], which describes quite well the behavior of $C_{A}(n)$ at small and intermediate "times" $n$ [37]. Applying Jensen inequality to Eqn. (17) one gets

$$
\frac{C_{A}(n)}{C_{A}(0)} \geq \exp \left(\log \left(1-C_{C}\right)\langle S(n)\rangle\right) \propto \exp \left(-C_{C}\langle S(n)\rangle\right)
$$


A rigorous calculation of the average $\langle S(n)\rangle$ in lattices of arbitrary spatial dimension was performed in [39]. Curiously, it turns out that in all dimensions $\langle S(n)\rangle$ exhibits the same $n$-dependence as the Smoluchowski constant in Eqns. $(10,13)$ integrated by time $n$ from 0 to $n$. Besides, all the numerical factors coincide. Slightly more involved analysis $[40,41,67]$ of the continuous-space analogue of $S(n)$ - the volume $V_{R}(t)$ explored by a diffusive spherical particle of radius $R$ during time $t$, has shown that, in all dimensions, the Smoluchowski constant is equal to

$K_{S m o l}(t)=\frac{d}{d t}\left\langle V_{R}(t)\right\rangle$

In the mathematical literature this volume is called "Wiener sausage". A realization of "Wiener sausage" is depicted in Fig. 3. Such a definition of the Smoluchowski constant is, probably, less convenient for the computation of $K_{S m o l}(t)$ in systems with conventional diffusion than its definition in Eqns. (8,9). However, it will be very useful for the explanation of results concerning reactions involving polymers transport properties of which are non-diffusive.

Therefore, particles concentrations can be represented as follows. For III and IIb the concentration decay is guided by

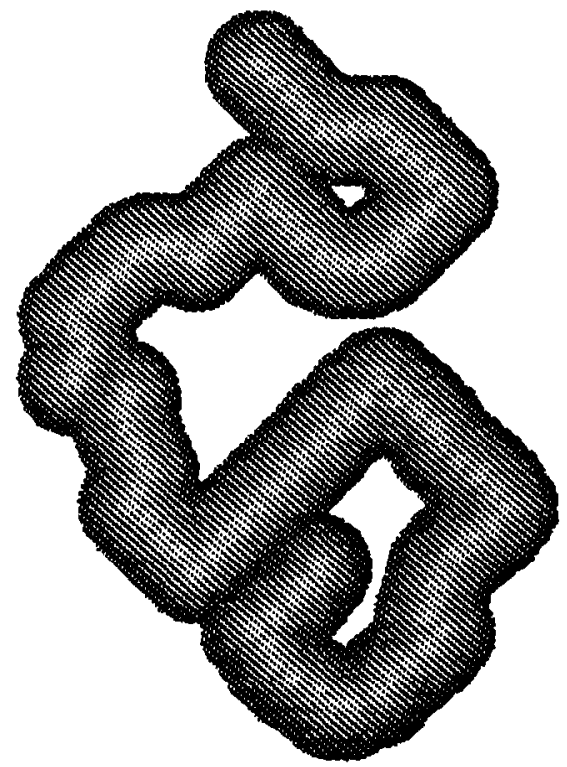

Fig. 3. Wiener sausage. Volume explored by a diffusive spherical particle of radius $R$ during time $t$. 
$C_{A}(t) \propto \exp \left(-C_{C}\left\langle V_{R}(t)\right\rangle\right)$

and for I and IIa one has

$C_{A}(t) \propto \frac{1}{\left\langle V_{R}(t)\right\rangle}$

Let us also note that since the Rosenstock approximation turns out to be equivalent to the Smoluchowski approach, one can say that Eqns. $(19,20)$ represent lower bounds on the decay laws, i.e. concentrations decay not faster than the dependences predicted by Eqns. $(19,20)$.

To close this section we would like to remark that for a long time the Smoluchowski approach was the basic starting point for the analytic treatments of kinetics in different chemical systems involving diffusive particles [1-6]. Subsequent works extended it in various directions, including the long-range potential interactions between particles of reactants $[14,42,43]$, examination of short and intermediate time corrections [1-6], especially in low dimensional systems, as well as the corrections caused by particle-solvent interactions and granularity of solvent [17-20]. The Smoluchowski approach has been also extended for the description of reaction kinetics in fractal systems [12,22,44-47] and reversible chemical reactions [48-]. However, the basic idea that one can use the space-variable independent "law of mass action" and calculate the rate constant using Fick's law with corresponding boundary conditions has always been kept. Therefore, it came as a great surprise that the Smoluchowski approach is in principle incorrect in describing the long-time kinetics of certain chemical reactions. The point is that for several reactions even small thermal fluctuations in particles concentrations drastically influence the long-time kinetics ultimately resulting in non-traditional behavior. In the next section we will consider two particular reactive systems with fluctuation-induced long-time behavior which cannot be understood and reproduced in terms of conventional approaches.

\section{EFFECTS OF SPATIAL FLUCTUATIONS ON THE REACTION KINETICS IN THE "SIMPLEST" CHEMICAL SYSTEMS}

In this section we examine the influence of fluctuations in particles concentrations on the kinetics of bimolecular reactions I-III taking place in the "simplest" chemical systems. 
2.1 Effects of fluctuations on the kinetics of recombination and binary reactions

Let us first analyze the influence of fluctuations on the kinetics of the recombination reaction IIa. Consider a reaction bath with particles $A$ and $\mathrm{B}$, presented at equal mean concentrations $C(0)$. Particles $\mathrm{A}$ and $\mathrm{B}$ diffuse and react when they approach each other at separation $R$. Suppose that initial spatial distributions of particles were perturbed by thermal fluctuations, so that fluctuations in particles concentrations are Gaussian and delta-correlated. Initial pairwise correlations (Eqn. (4)) are then given by

$$
P_{A A}(\lambda, 0)=P_{B B}(\lambda, 0)=C(0) \delta(\lambda) ; P_{A B}(\lambda, 0)=0
$$

We begin with some illustrative estimates of the reaction kinetics in such a system and then present more subsequent approaches.

Let us select in the reaction bath some spatial domain of linear size $\zeta$ and corresponding volume $\zeta^{d}$. In an initial state with equal mean concentrations of the two species, the numbers of A's and B's in this domain are, respectively,

$$
N_{A}(0)=C(0) \zeta^{d} \pm\left[C(0) \zeta^{d}\right]^{1 / 2}, N_{B}(0)=C(0) \zeta^{d} \pm\left[C(0) \zeta^{d}\right]^{1 / 2}
$$

where the first term stems from the contribution of the mean concentration and the \pm terms represent typical fluctuations in particle number in a volume $\zeta^{d}$.

Particle numbers in this domain evolve as follows. Until time $t_{\zeta} \propto \zeta^{2} / D$ only those $A$ and $B$ react which were initially placed in the vicinity of each other. At times $t$ greater than $t_{\zeta}$ one can expect complete diffusive mixing of particles within this domain. Since particles disappear in pairs, the first terms in the latter equations will cancel and one will have a rest of particles of one type, which were initially presented in excess. This excess concentration is proportional to $\left[C(0) \zeta^{d}\right]^{1 / 2}$. At $t=t_{\zeta}$ such a behavior occurs in every domain of size $\zeta$, and, therefore, the entire reaction bath at time $t_{\zeta}$ will be divided into domains $\zeta$ containing either A or B particles. Therefore, the mean concentration equals this local concentration,

$$
C_{A}(t)=C_{B}(t) \propto \frac{\text { fluctuation of number of particles in the domain }}{\text { volume of the domain }}
$$


As time evolves, greater and greater domains are involved since diffusion smooths inhomogeneities at larger and larger scales. One can assume that the typical domain's size grows with time as $\sqrt{D t}$. Substituting this scaling law into the latter equation one arrives at the following estimate [53-59]

$C_{A}(t)=C_{B}(t) \propto \frac{\left[C(0) \zeta^{d}\right]^{1 / 2}}{\zeta^{d}} \propto \frac{\sqrt{C(0)} t^{d / 4}}{t^{d / 2}} \propto \sqrt{C(o)} t^{-d / 4}$

This estimate of the typical behavior of particles concentrations due to spatial fluctuations indicates that in systems with spatial dimensionality $d$ less than 4 the decay of observables follows a slower time dependence as compared with the predictions of the formal kinetic scheme and the Smoluchowski approach. It means that spatial fluctuations in particles distributions become essentially important over long times and the description of the recombination reaction kinetics in terms of the law of mass action with the chemical rate constant or the Smoluchowski rate constant is incorrect.

Below we will present another approach to the description of the fluctuation-induced kinetics of recombination reaction (IIa), based on the analysis of the coupled equations describing the evolution of mean concentrations and hierarchy of correlation functions. As we have already mentioned, due to the non-linearity of the local reaction-diffusion equations (1-3) the evolution of the observables is coupled to the evolution of the pairwise correlations. The pairwise correlations are coupled to the third-order correlations. In turn, the latter are coupled to the fourth-order correlations and so on. Exact solution of this hierarchy is, of course, impossible and it has been clearly pointed out by earliest investigations [1-6]. However, one can devise an approximate method [54] for dealing with this hierarchy.

The system under consideration starts from the initial state which is characterized by Gaussian fluctuations. Diffusion of species can only smooth inhomogeneities and cannot give rise to the appearance of singularities in the fluctuation spectrum. Therefore, one deals with a system fluctuation spectrum which remains nearly Gaussian at all times. In view of this it seems natural to expect that, likewise for systems with Gaussian fluctuations, correlation functions of the fourth order decouple into the product of pairwise correlations. One can check that then the equations which govern the evolution of the third-order correlations have only trivial solutions, i.e. the latter are exactly equal to zero in the entire time 
domain $[60,61]$. Therefore, within such an assumption one gets an enclosed, with respect to the average concentrations and the pairwise correlations, system of differential reaction-diffusion equations, which can be solved analytically. Similar arguments for the evaluation of Eqn. (21) were invoked in [45]. Moreover, it was proved that such an approach leads to the definition of the rigorous upper bound on the mean concentration and this bound differs from the exact result only by a numerical factor [40].

The long-time forms of the pairwise correlation functions have been obtained in the case of equal particles diffusivities in [54] and in the case of unequal diffusivities in [62]. It was found that $P_{A B}(|\lambda|=R, t)$ in Eqn. (4) at times $t$ sufficiently large decreases diffusively

$$
\left.P_{A B}(|\lambda|)=R, t\right) \propto-\frac{C(0)}{\left(D_{+} t\right)^{d / 2}}
$$

Substituting (22) into (4) one arrives at the rate equation with the same structure as the "law of mass action" but with algebraically decreasing with time source term, which accounts for the evolution of the pairwise correlations. Due to the presence of the correlation-induced source term the long-time asymptotic solution of Eqn. (4) is no longer $C(t) \propto 1 / t$, but is governed by the decay of correlations [54],

$$
C(t) \propto \sqrt{-P_{A B}(\lambda=R, t)} \propto \sqrt{C(0)}(D t)^{-d / 4}
$$

i.e. is described by the asymptotic form in Eqn. (21).

Next we would like to make several remarks on the approach in [54]. At long times Eqn. (4) predicts a correct fluctuation-induced behavior of the observables. However, at intermediate times this approach leads to the formal kinetic scheme result irrespective of the relation between $K_{c h e m}$ and SC. This obvious shortcoming is caused by the approximation invoked for the enclosure of the reaction-diffusion equations. In fact, for small values of $\lambda$ the third order correlations are not equal to zero. It was shown in $[60,61]$ that small- $\lambda$ behavior of the third-order correlation functions is very important for the description of small and intermediate time behavior of the observables. Taking into account small- $\lambda$ behavior of the third-order correlations one arrives at the correct Smoluchowski-like renormalization of the reaction constant. Eventually, one obtains the modified rate equation of the form $[60,61]$ 
$\frac{d C(t)}{d t}=-\frac{K_{\text {Smol }} K_{\text {chem }}}{K_{\text {Smol }}+K_{\text {chem }}}\left[C^{2}(t)+P(|\lambda|=R, t)\right]$

which describes both the long and intermediate time behavior correctly. A similar approach, modified with respect to the physical conditions, was subsequently applied for the description of many-particle effects in the kinetics of reactions with different types of initial correlation spectra $[47,60,61]$ involving charged particles $[60,63]$, reversible chemical reactions [60-65] and reactions in fractal media [47,60].

Therefore, we have shown that the long-time kinetics of IIa are driven by the evolution of the pairwise correlations. The obtained results represent some new type of reaction kinetics mechanism: correlation-induced kinetics. In this sense the term "diffusion-controlled" kinetics becomes slightly meaningless and one should distinguish between "diffusion-controlled" kinetics in terms of the Smoluchowski approach and "diffusioncontrolled" correlation-induced kinetics.

It is worthwhile mentioning that "correlation-induced" kinetics are rather sensitive to the physical conditions. The correlation-induced slowing down of the recombination reaction kinetics is supported mainly by two different factors: sign of relevant correlation function (in the case under consideration observables are coupled to the A-B correlation function) and the rate at which these correlations decay. For IIa reaction the A-B correlations are negative, i.e. particles of unlike species tend to repel each other (in contrast, correlation functions of like species, say, A-A correlations, are positive, i.e. like species segregate). Since the time derivative of mean concentration (Eqn. (4)) is proportional to $-P_{A B}(t)>0$ the negative correlations slow down the decrease of mean concentration. Besides, it is very important that correlations decay diffusively, i.e. as $t^{-d / 2}$, and thus have higher "resistivity" with respect to reaction than the intrinsic rate and the Smoluchowski mechanism. It means that diffusion is an ineffective mechanism of mixing and fails to smooth spatial fluctuations. As a consequence, correlations control the elementary reaction act and thus the kinetics of the recombination reactions. However, if we consider systems in which, initially, particles A and B were distributed not independently of each other but presented in correlated $A B$ pairs we find completely different behavior. Pairwise A-B correlations are again negative, i.e. slow down the reaction kinetics, but do not change the kinetic law, since $P_{A B}(t)$ decays faster than diffusively, $P_{A B}(t) \propto-t^{-1-d / 2}$. Long-time solutions of Eqn. (4) are thus described by either the formal kinetic scheme or Smoluchowski approach (depending on the spatial 
dimensionality and the relation between rate constants) while correlations induce only vanishing with time corrections.

For the irreversible binary reaction I one can also evaluate the analogous closed reaction-diffusion equations, which account for the evolution of the pairwise correlations in the system. However, in contrast to the recombination reaction, the behavior of the mean particles concentration obeys the mean-field decay laws at all times. The reason for such a behavior is as follows: mean concentration of A particles is coupled to the A-A correlation function, which is always positive and thus can only accelerate the reaction kinetics. But, in this case, correlations decrease as $t^{-2-d / 2}$, i.e. at a slower rate than the square of the dependence in Eqn. (7). Therefore, mean-field theory prevails and correlations give rise only to the vanishing correction terms.

Let us also make an important remark that, within the descriptions in terms of conventional approaches - the formal kinetic scheme and the Smoluchowski approach, there is no difference in the kinetic behavior of the binary reaction and the kinetics of recombination reaction with equal initial concentrations. Both approaches predict the same type of time dependences for I and IIa. However, this distinction exists and stems from the behavior of correlations in the reactive system. For recombination reaction these correlations are decisive at long times and govern the long-time reaction kinetics, while for the binary reaction correlation effects are irrelevant.

\subsection{Effects of fluctuations on the kinetics of trapping reactions}

Trapping reaction III exhibits a fluctuation-induced behavior only in the particular case when traps are immobile, i.e. $D_{C}=0$, and randomly scattered in space [66-71]. Below we will present an estimate of fluctuation-induced asymptotics of $C_{A}(t)$ using the "optimal fluctuation" method.

Random spatial distribution of traps is characterized by essential fluctuations in the trap's local concentration. There are some regions with anomalously high concentration (greater than mean value $C_{C}$ ) and, at the same time, domains with anomalously low concentration of traps (Fig. 4). If one assumes Poisson distribution of traps in space the probability of having a spherical void (Fig. 4) of radius $\zeta$ with no traps at all will be given by

$P_{\text {void }}(\zeta) \propto \exp \left(-C_{C} \zeta^{d}\right)$ 


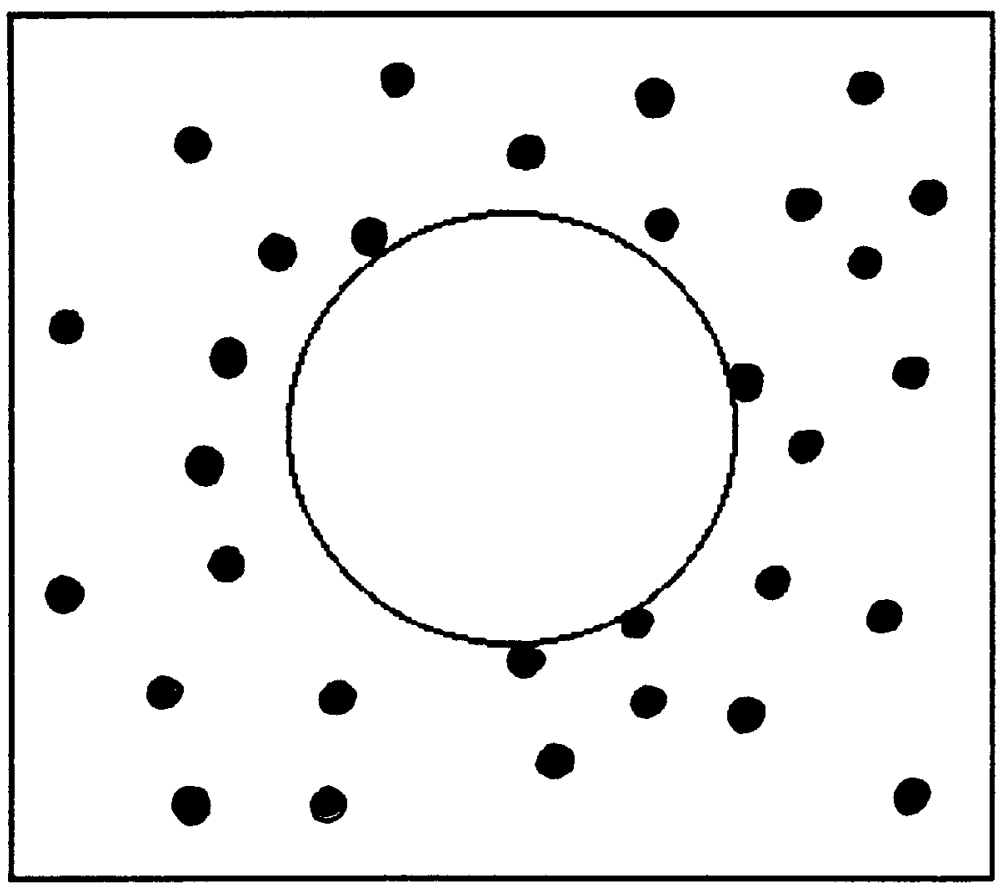

Fig. 4. Trap-free void in system with randomly placed immobile traps.

where the term in the exponent is equal to the mean number of traps in the void of size $\zeta$. Next, let us imagine one particle A which has occurred at zero time at the center of a trap-free void. The particle diffuses freely in such a void until it reaches the void's boundary. One can think that trapping occurs as soon as it reaches the boundary. The probability that this particle A survives until time $t$ is then the probability that the diffusive particle will remain inside the void until time $t$. The latter reads

$P(\zeta, t) \propto \exp \left(-D t / \zeta^{2}\right)$

Suppose next that one has some concentration of A particles. Apparently, at long times only those particles will survive that have appeared initially in sufficiently large trap-free voids. One can write the following estimate for the mean concentration of A particles,

$\frac{C_{A}(t)}{C(0)} \propto\left\langle P(\zeta, t) P_{\text {void }}(\zeta)\right\rangle$ 
where averaging extends over all voids, i.e.

$$
\frac{C_{A}(t)}{C(0)} \propto \int_{0}^{\infty} d \zeta \zeta^{d-1} \exp \left[-C_{C} \zeta^{d}-D t / \zeta^{2}\right]
$$

The integrand in the latter equation is a bell-shaped function and one can analyze the behavior of the integral using the steepest descent method. The maximum of the integrand is approached at $\zeta=\zeta^{*}(t)$ (void of "optimal size" at time $t$ ), which grows with time in proportion to $t^{1 /(d+2)}$. Replacing the integrand by the Gaussian centered at $\zeta^{*}(t)$ and integrating, one arrives at the decay form [66-71]

$$
\log \left(\frac{C_{A}(t)}{C(0)}\right) \propto-\left(\zeta^{*}(t)\right)^{d} \propto-C_{C}^{2 / d+2}(D t)^{d / d+2}
$$

which exhibits a slower time dependence than that predicted by the conventional approaches (Eqns. (11) and (15)). This behavior is dominated essentially by many-trap effects and cannot be understood on the basis of the mean-field theories.

\subsection{Estimates of the crossover times and reaction depths}

For $3 \mathrm{~d}$ reactive systems let us estimate the "crossover" time $t_{\text {cros }}$ from the mean-field behavior predicted by standard approaches to the fluctuation-induced asymptotic forms. We begin by estimating the time when the Smoluchowski dependence in Eqn. (12) is changed by fluctuation-induced asymptotic form (21). On comparing these decay laws we find that this time is of the order of $t_{\text {cros }} \propto R^{2} / D \varepsilon^{2}, \varepsilon=C(0) R^{3}$, i.e. is inverse proportional to the square of the initial volume fraction $\varepsilon$ of reactants and, thus, in diluted systems can be very large. The more informative parameter is the reaction depth which defines the concentration of particles present in the system at time $t_{\text {cros }}$. For the recombination reaction the reaction depth at time $t=t_{\text {cros }}$ is given by $C\left(t_{\text {cros }}\right) / C(0) \propto \varepsilon$. It means that an extremely small amount of species will survive until time $t_{\text {cros }}$, i.e. conventional approaches will correctly describe the reaction kinetics during a large time domain and, moreover, of the conversion of the bulk of reactive species. Only a very small amount of active particles will decay via the fluctuation-induced law in Eqn. (21). 
In the trapping problem the situation is even worse (from the viewpoint of many-particle effects). The crossover time depends algebraically on the volume fraction $\varepsilon_{C}$ of traps, $t_{\text {cros }} \propto R^{2} / D \varepsilon_{C}^{3 / 2}$ but the amount of particles A surviving until this time is exponentially small, $C\left(t_{\text {cros }}\right) / C(0)$ $\propto \exp \left(-\varepsilon_{C}^{-1 / 2}\right)$. To our knowledge, there is no experimental evidence of observation of anomalous fluctuation-induced laws in three-dimensional systems. In low-dimensional systems the crossover time is smaller and the amount of particles surviving until this time is greater than in three dimensions. The crossover from the dependences predicted by the conventional approaches to the fluctuation-induced asymptotics have been observed in numerical simulations $[44,45,55-58]$ and also experimentally [72-74].

Summing up the results presented in this section, we conclude that fluctuations, randomness and many-particle effects can influence significantly the kinetics of chemical reactions and lead to non-traditional behavior. The two particular cases we have mentioned are not the only examples of reaction schemes in which correlation effects are important. For example, recent studies of reversible reactions I-III have indicated that irrespective of the physical conditions - e.g. different initial relations between mean particles concentrations, charged or neutral reactive species, different types of initial correlation spectra - the long-time kinetics are governed by fluctuation effects. Mean-field considerations, based on the extensions of the Smoluchowski approach predict an exponential relaxation of particle concentrations to their equilibrium values. It was recognized that in contrast to the mean-field predictions, particle concentrations approach the equilibrium as a power-law $[21,60-65,75]$ as a consequence of fluctuation effects. Some alternative treatments of reversible reaction kinetics leading to the power-law long-time behavior are presented in $[28,76-79]$.

These fluctuation-induced deviations from the traditional behavior are, probably, of more theoretical than practical interest since they can only be observed at very large times when the bulk of reactive species is deactivated. However, as we set out to show in the remaining part of this paper, effects of such a type become extremely important from the earliest stages of reaction in systems in which particles execute subdiffusive random motion or the interparticle correlations are enhanced by the geometrical factors. 


\section{EXTENSIONS OF THE SMOLUCHOWSKI APPROACH TO THE REACTION KINETICS IN POLYMER SYSTEMS}

In this section we will consider extensions of the Smoluchowski approach to the kinetics of transport-controlled reactions in systems with polymers. In the first part of this section we will present results on the kinetics of bimolecular chemical reactions I-II involving active particles attached to passive polymers (Fig. 1). This analysis was first performed by de Gennes in his classic papers [31] on the kinetics of diffusion-controlled processes in dense polymer systems. In the second part we will extend the Smoluchowski-de Gennes approach for the description of homopolymerization reactions in systems with transport limitations. Finally, in the third part we will examine kinetics of trapping reactions III in systems with diffusive monomeric particles $A$ and polymerized traps (Fig. 2).

\subsection{De Gennes' approach to the kinetics of bimolecular reactions with participation of polymers}

Consider kinetics of chemical reactions involving chemically active groups which are attached to long flexible polymer chains (one active group per chain) in polymer solution (Fig. 1). Polymers migrate and thus active particles also migrate. Reaction between active particles occurs when they approach each other at separation $R$.

The first analytic description of reaction kinetics in such systems was proposed by de Gennes [31] who has reconsidered and generalized the Smoluchowski approach in order to incorporate the effects of polymers on the reaction process. He has shown that behavior of the Smoluchowski rate constant is controlled entirely by the form of random function $\left\langle X^{2}(t)\right\rangle$, which describes the mean-square displacement of an active group attached to a polymer chain. Here we will illustrate his results using a conceptually related approach based on Eqn. (18).

(a) Behavior of the Smoluchowski constant in case of non-diffusive random motion

In de Gennes' picture the crucial distinction between the reactions in the "simplest" chemical systems and the systems with active particles attached to polymer chains stems from the fact that transport characteristics of active groups involved are quite different. In the "simplest" chemical systems particles execute conventional diffusive motion with the mean square displacement growing in time as $\left\langle X^{2}(t)\right\rangle \propto t$. The motion 
of particles attached to flexible polymer chains is also random but has a more complicated nature than conventional diffusion and is dependent on different factors, such as the chain's length $L$, the chain's internal relaxation modes, quality of the solvent, concentration of polymers and entanglement effects. As a rule, the mean square displacement of a polymer segment, i.e. of an active particle, exhibits sublinear (subdiffusive) growth with time

$\left\langle X^{2}(t)\right\rangle \propto t^{\nu}, v<1$

for times $t$ less than $t_{c h a r} \propto L^{\beta}$, where the exponents $v$ and $\beta$ depend on the above-mentioned factors. Only at times $t$ greater than $t_{c h a r}$ does the motion of the active particle become diffusive, i.e. the diffusive law $\left\langle X^{2}(t)\right\rangle$ $\propto D(L) t$ is restored, with, however, the diffusion constant being a decreasing function of the chain's length $L$.

Let us estimate the volume $V_{R}(t)$ (Fig. 3) explored by a particle which executes random walk characterized by the mean square displacement in Eqn. (28). The only parameter that controls the behavior of this function is the value $\mu=d v / 2$. Depending on the value of $\mu, \mu>1$ or $\mu<$ 1 , the volume $V_{R}(t)$ exhibits two substantially different types of growth, connected with different statistics of returns (recurrency) of random walk executed by particle. In the first case, when $\mu>1$, the mean number of returns to the starting point (or any other point of the trajectory) tends to the constant value as time tends to infinity. If one examines how random walk with such properties fills the spherical volume $V_{X}(t)$ of radius $\sqrt{\left\langle X^{2}(t)\right\rangle}$ (Eqn. (28)), one finds that the volume of the Wiener sausage is essentially smaller than the volume $V_{X}(t)$, i.e. the particle explores only a small part of the volume $V_{R}(t)$. This type of motion was called by de Gennes "non-compact exploration". In the opposite case, when the parameter $\mu<1$, the mean number of returns to any point of the trajectory grows with time as $t^{1-\mu}$. Therefore, during time $t$ the particle visits nearly all the points in the volume $V_{X}(t)$, i.e. $V_{R}(t) \propto V_{X}(t)$. This case was called the case of "compact exploration". In the case of non-compact exploration $V_{R}(t)$ grows linearly with time. In the case of compact exploration $V_{R}(t)$ grows sublinearly with time, $V_{R}(t) \propto t^{\mu}$.

Employing Eqn. (18) one gets the following estimate for the time-dependence of the Smoluchowski constant in the cases of non-compact and compact explorations respectively

$K_{\text {Smol }}(t) \rightarrow$ constant, $t \rightarrow \infty, \mu>1$ 
$K_{S m o l}(t) \propto t^{\mu-1}, \mu<1$

Let us now return to the case of diffusive particles, considered in Section 1. In $3 d$ one has $\mu=3 / 2$ and, thus, the case of non-compact exploration with SC which tends to the constant value as $t \rightarrow \infty(10,29)$. In $1 \mathrm{~d}$ systems the value of $\mu$ is less than unity and we have the case of compact exploration with SC being the decreasing function of time. From the results of Section 1 one can infer that in the marginal case $\mu=1,\left(d_{m}\right.$ $=2 / v$, one will have the logarithmic decrease of SC. Similar ideas, but otherwise stated, were invoked for the description [44-47] of bimolecular reactions taking place in fractal systems. In such systems, characterized by non-integer spatial dimension $D_{f}$, the random motion of particles is characterized by anomalous diffusion law, similar to that in Eqn. (28). In

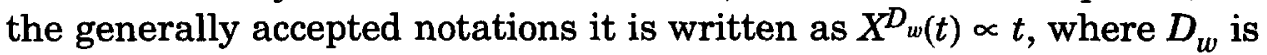
the so-called fractal dimension of random walk. For the conventional diffusive motion taking place in systems with integer dimensionality $D_{w}$ $=2$. An analysis of SC behavior leads to Eqn. (30) with $\mu=D_{f} / D_{w}, d_{m}=$ $D_{w}$. This dependence has been confirmed by numerical simulations of the reaction kinetics taking place in fractal systems $[12,44,45]$.

Finally, let us note that anomalous behavior of SC in systems with $\mu$ $\leq 1$ is also the consequence of fluctuations in spatial distributions of reactants [80]. However, these fluctuations have quite a different nature compared with the fluctuation states considered in Section 2. Below we will illustrate this statement using the same type of arguments which were employed for evaluation of the estimate in Eqn. (21). Consider a $d$-dimensional system with binary reaction, $A+A \rightarrow P$, involving particles A which execute random walk characterized by Eqn. (28) and react when they meet each other with unit probability $\left(K_{\text {chem }}=\infty\right)$. Consider next in the reaction bath some spherical region of radius $\zeta$ and volume $\zeta^{d}$ containing some amount of particles A. At time $t_{\zeta} \propto \zeta^{2 / N}$ one can expect a complete mixing of particles $A$ in this region. Therefore, at time $t_{\zeta}$ all particles A presented in this region will react. Therefore, there will be no particles $A$ if the initial number of particles was odd and a single $A$ particle, if this number was even. At time $t_{\zeta}$ such a behavior occurs in every region of size $\zeta$. Thus, particle concentration will be $C \propto 1 / \zeta^{d}$. If spatial dimensionality $d$ is less than $d_{m}=2 / v$ the size $\zeta$ will grow with time as $\zeta \propto t^{v / 2}$, and, therefore, $C(t) \propto 1 / t^{d v / 2}$. The Smoluchowski constant is the derivative $K_{S m o l}(t)=d(1 / C(t)) / d t \propto t^{\mu-1}$, i.e. one recovers the result in Eqns. (30). Thus, the fluctuation states which support an anomalous behavior of particles concentrations and the Smoluchowski constant in 
systems with $\mu \leq 1$ are the fluctuations of the odd-even number of particles in certain volumes.

\section{(b) Rouse and reptation models of polymer dynamics}

Let us now discuss the possible values of the parameter $v$ in the case of reactions involving active particles attached to warmlike polymer chains. In [31] de Gennes has considered two models of polymer chain dynamics - the Rouse model [81] and the reptation model [82-84].

In the Rouse model the polymer chain of length $L$ is represented as a set of $N$ beads successively connected by harmonic springs. The mean distance between beads is equal to $l, L=l N$. Each bead is subject to uncorrelated random forces (Langevin source) which mimic the effect of solvent on the chain's dynamics. If one tags one bead of a Rouse chain, e.g. by placing an active group, and follows its motion, the observed behavior of the mean square displacement will be as follows. For times $t$ less that $t_{\text {char }} \propto L^{2}, \beta=2$; the mean square displacement will be governed by Eqn. (28) with $v=1 / 2$. For times greater than $t_{\text {char }}$ the mean-square displacement will be described by diffusive law, i.e. by Eqn. (28) with $v=$ 1 and $L$-dependent diffusive constant. Combining these expressions and Eqns. $(29,30)$ one obtains the following results,

$K_{S m o l}(t) \propto t^{-1+d / 4}, t \leq t_{c h a r}$

and at times greater than the characteristic time SC is defined by Eqns. $(10,13)$ with, however, the reaction radius equal to the polymer's gyration radius, $R \propto L^{1 / 2}$, and the diffusion constant $D(L) \propto L^{-1}$. Therefore, the Smoluchowski-de Gennes approach predicts that the steady state SC decreases with $L$ as $1 / \sqrt{L}$.

Therefore, one finds that in systems with particles of reactants attached to Rouse polymers the reaction kinetics follows an anomalous behavior within a large time domain $t<t_{c h a r} \propto L^{2}$. This behavior is described* by Eqns. $(19,20)$ with $V_{R}(t) \propto t^{d / 4}$. At long times, i.e. times greater than $t_{\text {char }}$ the decay of particle concentrations is described by the dependences in Eqns. $(11,12,14,15)$ with $D \propto 1 / L$ and $R \propto L^{1 / 2}$.

* The intermediate time behavior of I and IIa described by Eqn. (20), $C_{A}(t) \propto 1 / t^{d / 4}$, is specific for bimolecular reaction involving active particles attached to Rouse polymers and should not be confused with the fluctuation-induced long-time dependence in Eqn. (21), describing IIa reaction involving monomeric particles. Equation (20) is essentially mean-field result and the coincidence of its functional form with Eqn. (21) is accidental. 
The second case considered in [31] is the model of reptation - an approximate description of the dynamics of a polymer chain in the presence of fixed randomly placed obstacles. The intrinsic properties of the polymer are represented by the Rouse model, i.e. beads connected by harmonic springs and being under the action of Langevin delta-correlated source. The obstacles impose topological constraints on the chain's motion - the chain can cross neither of them. This model was formulated by de Gennes [82] and was subsequently applied to melts and solutions by Doi and Edwards [85]. The reptation picture of the chain's motion is based on the following consideration. For a given conformation of a polymer one can draw a primitive path, i.e. the shortest path connecting the two ends of the chain with the same topology as the chain itself relative to the obstacles. The motion of the chain in the direction perpendicular to the primitive path is strongly hindered by obstacles and occurs primarily along the primitive path with new regions explored only by the ends of the chain. Such a type of polymer chain motion was called "reptation". The reptation picture predicts the following behavior of the tagged bead of a polymer chain. At the intermediate time $\left(t<t_{c h a r}=L^{3}\right.$, i.e. is greater than that in the Rouse model) the bead's mean square displacement is guided by Eqn. (28) with $v=1 / 4$. At times greater than $t_{c h a r}$ the conventional diffusive behavior $(v=1)$ is established with the diffusion coefficient $D(L) \propto 1 / L^{2}$.

Therefore, if one considers the reaction kinetics in systems with reptation motion of polymers, one will obtain the following results for the Smoluchowski constant

$K_{S m o l}(t) \propto t^{-1+d / 8}, t \leq t_{c h a r}$

and Eqns. $(10,13)$ (with $D \propto \mathrm{L}^{-2}$ and $R \propto L^{1 / 2}$, i.e. in three dimensions $K_{\text {smol }} \propto L^{-3 / 2}$ ) for times greater than the characteristic time. Consequently, the reaction kinetics at times $t<t_{c h a r}$ will be governed by Eqns. $(19,20)$ with $V_{R}(t)$ growing as $t^{d / 8}$. At long times the decay of particles concentrations will be governed by dependences in Eqns. (11-15) with $D$ $\propto L^{-2}$ and $R \propto L^{1 / 2}$.

Considered models of polymer dynamics are quite realistic. Measurements of tracer diffusion coefficients and of viscosities (e.g. [86]) in melts of polymers with a sufficiently low value of $L$, as well as numerical simulations $[87,88]$, show molecular weight dependences and the intermediate times dynamics of polymers that are consistent with predictions of the Rouse model. The reptation picture has proven to be highly successful in describing the dynamics of entangled flexible chain poly- 
mers in concentrated solutions of sufficiently long chains [89]. On the other hand, some recent numerical and analytical analysis of polymer dynamics in porous media indicate [88] the appearance of some deviations from the predictions of these models. It was found that the maximal relaxation time ( $t_{c h a r}$ in our notations) grows with $L$ at a faster rate than is predicted by the reptation model, $t_{\text {char }} \propto L^{2}$. It was conjectured [90] that this dependence can be even exponential strong due to effects of temporal trapping of polymers in random cavities with anomalously low porosity. Consequently, the dependence of the diffusion constant on the chain's length can be stronger and the mean square displacement smaller than is predicted by the Rouse and the reptation models. This question is not yet completely understood and requires a careful experimental or analytical analysis. In any case, the Smoluchowski-de Gennes approach is quite general and is not restricted to these particular models. Therefore, experiments on the reactions kinetics in systems with polymerized active particles can serve as an additional tool for characterizing polymer dynamics in disordered media.

To conclude this section, we have illustrated the results of the Smoluchowski-de Gennes approach to the kinetics of bimolecular reactions involving active particles, which do not diffuse freely in the reaction bath but are attached to polymers in polymer solution. We have shown that this approach predicts an anomalous kinetic behavior as compared with the kinetics in the simplest chemical systems. At the intermediate times this anomalous behavior is associated with the internal relaxation times of the macromolecules and, as a consequence, with non-diffusive random motion of reactants. Let us emphasize the crucial point that in the case of "compact exploration" molion the rale constant is independent of the reaction radius and decreases with time. Therefore, as we have mentioned in Section 1, the behavior of the apparent rate constant is controlled only by random transport of the reactive species to each other and the chemical rate is irrelevant. This is the reason for inferring [31] that kinetics of bimolecular reactions involving particles attached to polymers are transport-controlled. Within the large- $t$ limit, i.e. at times greater than the maximal relaxation times of the macromolecules, in $3 \mathrm{~d}$ systems rate constants approach steady state values. However, these values are inversely proportional to the powers of polymer lengths $L$ and, therefore, $K_{S m o l}$ can be essentially smaller than chemical rate $K_{\text {chem }}$ and hence control reaction kinetics. The decay laws at large times are characterized by Smoluchowski-like time dependences in Eqns. $(11,12,14,15)$ with $L$-dependent reaction radius and diffusion constant. 
3.2 Polymerization reactions in systems with transport limitations

The polymerization process is described by the following reaction scheme

$A_{N}+A_{M} \rightarrow A_{N+M}$

i.e. is the set of parallel bimolecular binary reactions involving different molecules $A_{N}$, where $A_{N}$ stands for a polymer of the molecular weight $N$ (an $N$-mer).

The state of the system is characterized by the molecular weight distribution (MWD), $P(N, t)$. This property defines the probability (or concentration) of having an $N$-mer at time $t$. The temporal evolution of $P(N, t)$ is guided by the kinetic coagulation equation

$\frac{\partial P(N, t)}{\partial t}=\frac{1}{2} \sum_{I+J=N} P(I, t) P(J, t) K(I, J)-P(N, t) \sum_{J=1}^{\infty} P(J, t) K(N, J)$

where $K(I, J)$ is the overall rate at which an $I$-mer reacts with a $J$-mer.

The form of $K(I, J)$ is controlled by two factors. One of them is the chemical rate, which defines the elementary reaction act - synthesis of a large macromolecule from two smaller polymers being at contact. This rate is dependent on the particular details of the process-activation barrier, molecular weights of polymers and a number of active groups in a polymer. The second factor stems from the transport limitations - in order to react polymers have to meet each other within a random migration process.

The effects of transport limitations on the kinetics of polymerization reactions have been the focus of interest for a long time. In [91-93] it was mentioned that the behavior of $P(N, t)$ in systems with randomly movable polymers must be quite different to that in systems with perfectly stirred macromolecules. In systems with transport limitations smaller molecules migrate at a faster rate than the larger ones and thus have a higher probability of reacting. This must result in a depletion of small molecules in the system. For very long chains again there is a deficit because their diffusivities are very small. Therefore, it is expected that the MWD is a bell-shaped function of the molecular weight $N$ centered around the most probable polymer weight $N(t)$, which grows with time. In this chapter we explore the Smoluchowski-de Gennes approach and an idea of "compact 
exploration" motion of active groups involved, in order to obtain the kinetic description of homopolymerisation reactions in $3 \mathrm{~d}$ systems with transport limitations. We set out to show that within such an approach the forms of $P(N, t)$ can be found analytically [94].

Using the results of the Smoluchowski-de Gennes approach, we assume that the influence of polymer transport on the polymerization kinetics can be captured by introducing to the Smoluchowski constant the parameters that are specific for polymers, i.e. we stipulate that the Smoluchowski constant has its usual form in Eqn. (10), i.e. $K_{S m o l}(I, J) \propto$ $R(I, J) D(I, J)$, where $D(I, J)$ is the sum of diffusion coefficients of an $I$-mer and a $J$-mer, $D(I, J)=D(I)+D(J)$; and the reaction radius $R(I, J)$ is the sum of polymers' gyration radii, $R(I, J)=R(I)+R(J), R(J) \propto J^{z}$. Such an assumption is also supported by recent calculations of the Smoluchowski constant in a system of long rod-like molecules [93]. As we have seen from the Rouse and reptation models of polymer dynamics, the diffusivity of a polymer is a decreasing function of its molecular weight. In our consideration we stipulate that it is either an algebraic function, $D(I) \propto I^{-p}$, with $p>z$, or a stretched-exponential function [90], $D(I) \propto \exp \left(-\varepsilon I^{q}\right)$ with $q>0$.

Correspondingly, the Smoluchowski constant depends on $I$ and $J$ as

$K_{S m o l}(I, J) \propto f_{I}+f_{J}$

with

$f_{I}=I^{-\alpha}, \alpha=p-z>0$

or

$f_{I}=\exp \left(-\varepsilon I^{q}\right)$

Let us next estimate the resistivity of the elementary act and transport of polymers to each other. Suppose that the system under consideration starts from the initial monodisperse distribution,

$P(N, t=0)=\delta_{1, N}$

where $\delta_{1, N}$ is the Kroneker delta, i.e. initially only monomeric particles are present in the system. Suppose next that for monomers $K_{\text {chem }}(1,1)<<$ $K_{S m o l}(1,1)$. As time evolves small molecules react and form larger ones, so that there appears some typical size of the macromolecules $I(t)$ corre- 
spondent to the time scale. Let us note that the Smoluchowski constant decreases with an increase of the polymer's weight, while the chemical constant under most conditions increases with an increase of the molecular weight. It means that after some time $t^{*}$, such that $K_{c h e m}\left(I\left(t^{*}\right), I\left(t^{*}\right)\right)=$ $K_{\text {Smol }}\left(I\left(t^{*}\right), \mathrm{I}\left(t^{*}\right)\right)$, the transport of macromolecules to each other will gain a progressively higher resistivity than the chemical rate, i.e. kinetics of polymerization will be controlled by the Smoluchowski constant and thus transport-controlled. Therefore, we stipulate that polymerization kinetics, at sufficiently large times, are described by Eqn. (33) with

$K(I, J)=K_{\text {Smol }}(I, J)$

given by either Eqn. (34) or by Eqn. (35).

The solutions of equations similar to Eqn. (33) have been analysed using scaling arguments in [95,96] for algebraically decreasing kernels in Eqn. (34). However, it turns out [94] that Eqn. (33) can be solved explicitly for arbitrary kernels $f_{I}$, which decrease with an increase of $I$. It was found [94] that the MWD $P(N, t)$ has the multinomial form

$P(N, t)=-M_{0}(t) \sum^{\prime} \frac{\Gamma_{1}^{a_{1}} \Gamma_{2}^{a_{2}} \ldots \Gamma_{N}^{a_{N}}}{a_{1} ! a_{2} ! \ldots a_{N} !}$

where the prime means that the summation extends over all possible integer and positive solutions $\left\{a_{N}\right\}$ of the Diophant equation

$1 a_{1}+2 a_{2}+3 a_{3}+\ldots+N a_{N}=N$

and the function

$\Gamma_{J}=-\frac{\exp \left[-T(t) f_{J}\right]}{J}$

The time scale $T(t)$ is given by $T(t)=\int_{0}^{t} M_{0}(t) d t, M_{0}(t)$ being the zeroth moment of the MWD

$M_{0}(t)=\sum_{N=1}^{\infty} P(N, t)$

The evolution of $M_{0}(t)$, in turn, is governed by the closed non-linear equation 
$\frac{\partial M_{0}(T)}{\partial T}=-M_{0}(T) \int_{0}^{\infty} d x F(x)\left\{1-\exp \left[-\sum_{J=1}^{\infty} \frac{\exp \left(-J x-T f_{J}\right)}{J}\right]\right\}$

where $F(x)$ and $f_{J}$ are related to each other by Laplace transformation

$f_{J}=\int_{0}^{\infty} d x \exp (-J x) F(x)$

The large- $T$ solutions of Eqn. (38) can be found as follows. For the algebraic dependence in Eqn. (34) one has asymptotically

$\frac{d M_{0}(T)}{d T} \propto-\frac{M_{0}(T)}{\alpha T}$

This differential equation can be integrated, $M_{0}^{-\alpha}(t) \propto \int_{0}^{t} d t M_{0}(t)$, and yields,

$M_{0}(t) \propto t^{-1 /(1+\alpha)}$

This is exactly the result obtained by means of scaling arguments in $[95,96]$. Let us also examine the form of the MWD in the limit of small molecular weights $N$ (or large times). The large- $T$ behavior of $P(N, T)$ is supported by the term with the smallest eigenvalue, i.e. by the solution $\left\{a_{N}\right\}$ of the Diophant equation with the configuration $a_{k}=0, k=1,2, \ldots$, $N-1 ; a_{N}=1$. For this, $P(N, T)$ reads,

$P(N, T(t))=M_{0}(t) \frac{\exp \left[-T(t) N^{-\alpha}\right]}{l}$

At large- $t$ limit, $T(t) \propto t^{\alpha /(1+\alpha)}$, the MWD takes the scaling form and can be expressed as a function of only one variable $X=N M_{0}(t)$. For $X<<1$, the solution of Eqn. (33) is the following bell-shaped function

$P(N, t)=M_{0}^{2}(t) \frac{\exp \left(-X^{-\alpha}\right)}{X}$

This result also coincides with the scaling prediction $[95,96]$. 
For the case of purely exponential kernels (Eqn. (35) with $q=1$ )

$$
\frac{d M_{0}(T)}{d T} \propto-\frac{M_{0}(T)}{T \log (T)}
$$

Being integrated it yields the following relation between $M_{0}(T)$ and $T$, $M_{0}(T)=1 / \log (T)$, and, consequently, entails the following large- $t$ behavior

$$
M_{0}(t) \propto \frac{1}{\log (t)}
$$

Finally, for stretched-exponential kernels we obtain

$M_{0}(t) \propto \frac{1}{\log ^{1 / q}(t)}$

Therefore, we have shown that transport limitations can influence dramatically kinetics of polymerization reaction. They slow down the decay of the concentration of different polymers, $M_{0}(t)$, as compared with the systems without transport limitations* and thus slow down growth of the average polymer size. Besides, the presence of transport limitations results in the change of functional form of the molecular weight distribution: it becomes a bell-shaped function of $N$, in contrast to the monotonic dependence in systems without transport limitations.

\subsection{Trapping reactions in polymer systems. Mean-field approach}

Consider a frozen polymer solution in which immobile polymers are presented at mean concentration $n_{c h}$. Each polymer of length $L$ contains $N$ traps $C$. The mean distance between particles along the chain is $l=$ $L / N$. We assume that $N$ is sufficiently large so that spatial distribution of particles $C$ follows the spatial distribution of polymer chain segments, i.e. if the polymer's gyration radius scales with chain length $L$ as $\left\langle R_{g}^{2}(L)\right\rangle \propto L^{2 z}$ it scales also with $N$ as $\left\langle R_{g}^{2}(N)\right\rangle \propto N^{2 z}$. Suppose next that one has some concentration of monomeric particles $A$, which diffuse freely and are annihilated as soon as they approach at distance $R$ any trap $C$ placed at any polymer (Fig. 2). Here we will examine the kinetics of trapping reactions in such systems in terms of a mean-field

* $K(I, J)$ is nonvanishing function of $I$ and $J$ and $M_{0}(t)$ decays not slower than $1 / t$. 
Smoluchowski-like approach. Let us assume that concentration of polymers is small enough so that polymers do not overlap and one can neglect screening of traps placed on one polymer by traps placed on other polymers. Then, following Smoluchowski, we write the mean concentration of particles $A$ as

$$
\frac{C(t)}{C(0)}=\exp \left(-n_{c h} \int^{t} d t K_{S m o l}(N, t)\right)
$$

where $K_{S m o l}(N, t)$ is the rate at which diffusive particles $A$ react with a single polymer chain containing $N$ absorbing beads $C$. To calculate this rate constant we employ the usual Smoluchowski scheme in Eqns. (8) with the modified absorbing boundary condition in Eqn. (8b). In case of $N$ traps this boundary condition has zero probability of finding a particle A at the surface $S\left(R_{i}\right)$ of any $i$ th trap,

$$
P\left(r \subseteq \sum_{i=1}^{N} S\left(R_{i}\right), t\right)=0
$$

The rate $K_{S m o l}(N, t)$ is then defined as the sum of fluxes through $N$ reaction spheres

$$
K_{S m o l}(N, t)=D \sum_{i=1}^{N} \int_{\left|r-r_{i}\right|=R} \nabla P(r, t) d S\left(R_{i}\right)
$$

where $r_{i}$ is the radius-vector of $i$ th trap.

Two different approaches to the solution of diffusion equation with absorbing boundary conditions on surfaces of $N$ traps arranged in polymer chain have been proposed $[97,98]$. The first is based on the randomphase approximation and the second on the renormalization group analysis. Both of them lead to the same result for the rate constant. It was found that $[97,98]$

$$
K_{\text {Smol }}(N, t \rightarrow \infty) \propto R D N^{1 / 2}
$$

i.e. it occurs that for traps arranged in a polymer chain the Smoluchowskilike rate constant grows at a rate that is sublinear in $N$. It means that 
correlations in trap placement induce strong screening effects. Curiously enough, this result contradicts apparently quite reasonable intuitive arguments. Namely, the mean radius of coil grows with $N$ as $\sqrt{N}$, and correspondingly, the mean concentration of traps (segments) drops with $N$ as $N^{1-d / 2}$, i.e. as $1 / \sqrt{N}$ in three dimensions. Therefore, it seems natural to expect that traps do not screen each other, and the overall rate constant $K_{S m o l}(N, t)$ is the SC in Section 1 multiplied by $N$. However, it occurs that the correlations in the trap placement lead to strong screening which has the effect of drastically decreasing the rate constant, i.e. enhancing the survival probability of particles $A$.

For non-Gaussian chain conformations with $z \neq 1 / 2$ it was found that [97]

$K_{S m o l}(N, t \rightarrow \infty) \propto N^{z}$

i.e. is simply proportional to the radius of gyration. Therefore, it occurs that a polymer chain containing $N$ traps is effectively one large trap of radius $N^{z}$.

We close this part with an estimate of time-dependent corrections to the Smoluchowski-like rate constant in Eqn. (43). It was found that in the case of a Gaussian polymer chain the Smoluchowski-like constant has the usual form

$K_{S m o l}(N, t) \propto 4 \pi R D N^{1 / 2}\left[1+\frac{R N^{1 / 2}}{(\pi D t)^{1 / 2}}+\ldots\right]$

with the only difference that the time-dependent correction term is proportional not to the trap's radius $R$, but to a much greater value the effective radius of a polymer chain. Thus, in contrast to the systems with monomeric traps, one can expect the appearance of the kinetic stage described by stretched-exponential pattern [97]

$$
\frac{C(t)}{C(0)} \propto \exp \left(-n_{c h} N R^{2 \sqrt{D t})}\right.
$$

which will be valid until times of order $R^{2} N / D$. 


\section{INFLUENCE OF FLUCTUATION EFFECTS ON THE REACTION KINETICS IN POLYMER SYSTEMS}

\subsection{Kinetics of trapping reactions in systems of polymerized traps}

In this section we will reconsider from the viewpoint of many-chain effects the model in (3.3) - trapping reaction in the presence of polymerized traps (Fig. 2). Here the concentration of polymers is not assumed to be small and the chains can overlap each other. In our analysis we will extend simple estimates of Section 2, based on the optimal-fluctuation method, to the system under consideration.

(a) Calculation of the distribution function of polymer-free voids in a frozen polymer solution

We begin with the calculation of the void (chain-free) size distribution [99] in a solution of frozen polymer chains (Figs. 5 and 6), presented at mean concentration $n_{c h}$. Each chain contains $N$ beads and has a radius of gyration $\left\langle R_{g}^{2}(N)\right\rangle \propto N^{2 z}$.

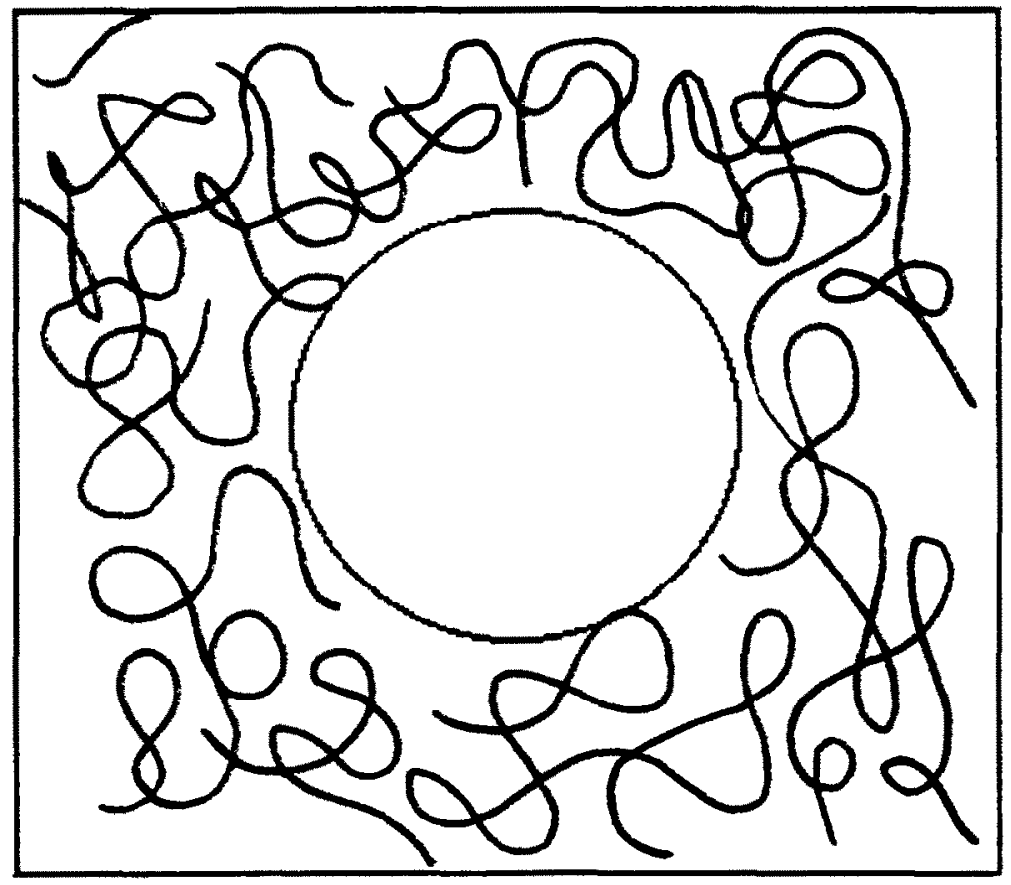

Fig. 5. Random polymer-free void in polymer solution. 


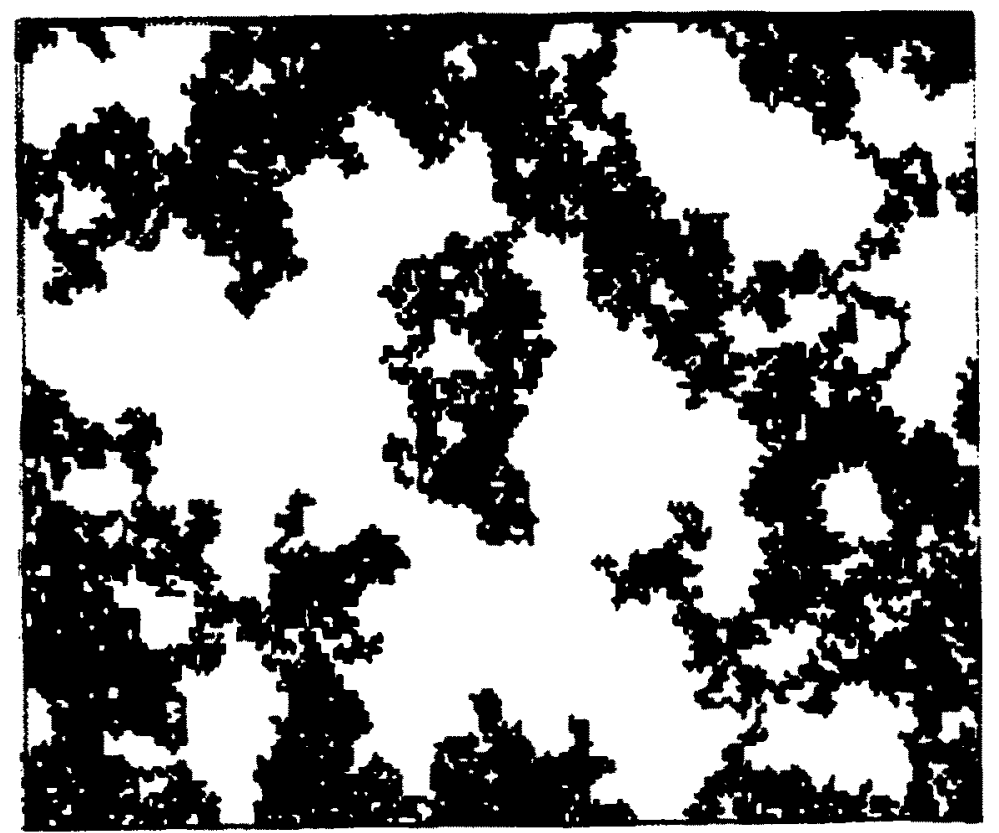

Fig. 6. Lattice picture of frozen polymer solution. This realization was built as follows: 30 lattice sites were chosen at random. Next, from each of these sites we have "grown up" freely-jointed chains (random walk trajectories) containing 3000 segments each. No excluded volume effects were taken into account. Polymer substrate occupies 40 per cent of lattice sites.

The probability $P_{\text {void }}(\zeta, N)$ of having a polymer-free void of radius $\zeta$ can be written formally as a product of two components

$P_{\text {void }}(\zeta, N)=P_{\text {end }}(\zeta) P_{\text {sur }}(\zeta, N)$

where the first multiplier is the probability of having no chain ends in the void. For mutually-independent chains the distribution of their ends is Poisson and one has for $P_{\text {end }}(\zeta)$ the usual negative exponential form similar to that in Eqn. (24), $P_{\text {end }}(\zeta) \propto \exp \left(-n_{c h} \zeta^{d}\right)$. The second multiplier in (45) is more complicated. It defines the conditional probability that neither chain of length $N$, with its end being randomly distributed outside the void, crosses the void's surface. The precise form of this function can be understood if we formulate its definition in a slightly different fashion. Let us first consider the case of Gaussian chains.

Suppose that one has a single immobile sphere of radius $\zeta$ placed in 
the "sea" of infinitesimal particles $\mathrm{C}$, distributed initially with some mean concentration $n_{c h}$ outside the sphere. Particles $\mathrm{C}$ diffuse with diffusion coefficient $l^{2} / 2 d$. Let us next suppose that this sphere is a particle $\mathrm{A}$ and it disappears as soon as any of the $C$ particles reach its surface. In the literature on chemical kinetics this problem is called target reaction (see, e.g. Ref. [37]) and it is well known that the Smoluchowski approach entails an exact result for the survival probability of the target particle. One can see that the probability that neither of the Gaussian polymers of length $l N$ presented at mean concentration $n_{c h}$ crosses the surface of the spherical volume of radius $\zeta$ and the survival probability of the target particle of radius $\zeta$ in the presence of diffusive traps are equivalent to each other. Therefore, we can infer that [99]

$$
P_{s u r}(\zeta, N)=\exp \left[-n_{c h} \int_{0}^{N} K_{S m o l}(N) d N\right]
$$

where $K_{\text {Smol }}(N)$ is usual SC in Eqns. $(10,13)$ (up to the change of notations $t \rightarrow N$ and $R \rightarrow \zeta$ ). Therefore, for 3d solutions of frozen Gaussian chains the probability of having a polymer-free void of radius $\zeta$ is equal to

$$
P_{\text {void }}(\zeta, N)=\exp \left(-n_{c h} \zeta^{d}-n_{c h} l^{2} \zeta N\right)
$$

Let us stress several important features of the obtained expression (47). It shows that on large scales $(\zeta \geq \sqrt{N})$ void-size distribution is a negative exponential, i.e. on large scales the polymer solution can be viewed as a Poisson ensemble of immobile point particles, presented at mean concentration $n_{c h}$. In contrast, below the correlation length $l N^{1 / 2}$ the distribution is fractal. If one compares the probability of having a void of radius $\zeta$ in polymer solution with $n_{c h} N$ beads and the same probability in a system with Poisson distribution of free beads, presented at the same mean concentration $C_{C}=n_{c h} N$, one finds that this probability is much greater in polymer solutions. It can be seen, for example, from our Fig. 6 which represents typical realizations of Gaussian polymers on a two-dimensional surface. Due to the "interconnection" of traps into polymer chains the polymer-free voids are much more pronounced than in systems with random placement of independent point traps. In the next section we will see how this circumstance affects the kinetics of trapping reactions in polymer solutions. 
Similar calculations are also possible for two-dimensional systems of Gaussian chains. In 2D solutions of Gaussian polymers the void-size distribution has a more complicated form* and was analyzed in [100].

To analyze the forms of the void-size distribution in systems with arbitrary chain conformations, where the exponent $z$ is not necessarily equal to $1 / 2$, we again employ the Smoluchowski-de Gennes approach elaborated for the description of the reaction kinetics with non-diffusive random motion of reactants (here $2 z$ is an analogue of the dynamic exponent v), i.e. assume the validity of Eqn. (18) and represent $\int{ }^{N} K_{S m o l}(N) d N$ as the volume visited by a spherical particle of radius $\zeta$ during time $N$. For polymers in a coil phase we have the case of non-compact exploration, since $d z$ is always greater** than 1 , and thus the Smoluchowski-like constant $K_{S m o l}(N)$ will approach some $N$ independent value for $N$ sufficiently large and will depend essentially on $\zeta$. Making use of the blob-like estimate in [99] or Eqn. (III.23) in [31], $K$, one obtains the following dependence

$$
\int_{0}^{N} K_{S m o l}(N) d N \propto N l^{1 / z} \zeta^{d-\frac{1}{z}}
$$

and, consequently, the void-size distribution can be written as (for $d z>$ 1)

$$
P_{\text {void }}(\zeta, N)=\exp \left[-n_{c h} \zeta^{d}-n_{c h} N l^{1 / z} \zeta^{d-\frac{1}{z}}\right]
$$

To close this part let us make several remarks on the distribution function in Eqn. (48). The derivation of Eqn. (48) is based on the uncontrollable and rather questionable assumption that polymers are mutually-independent. In principle, the repulsion between coils or excluded volume effects might change its functional form. However, Eqn. (48) reproduces several results well-known for real polymer systems. For instance, let us estimate the average void-size (or the correlation length) in the case of very concentrated polymer solutions, when the volume

* The result in Eqn. (13) is useless since it corresponds to the limit of very small voids, $\zeta \ll \sqrt{l^{2} N}$. We are interested in the behavior of the 2D Smoluchowski constant at the entire "time" domain $N$.

** Except the case of Gaussian polymers in two-dimensional systems when $d z=1$. 
fraction of polymers is high $n_{c h} l^{3} N \propto 1$. In accord with the Flory theorem $R(N) \propto N^{1 / 2}$, i.e. $z=1 / 2$, and one then obtains from the normalized Eqn. (48) $\langle\zeta\rangle \propto 1 / n_{c h} l^{3} N$. This is the correct result for the correlation length in polymer melts $[83,84]$. Consider next the case of semidilute solutions, $C^{*}<n_{c h} l^{3} N<1, C^{*}$ is the concentration at which chains begin to overlap each other. In semidilute solutions chains are swollen and are characterized by $R(N) \propto N^{z}$ with $z=3 /(d+2)$ being the Flory exponent. Equation (48) predicts that in $3 d$ solutions the average void size behaves as $\langle\zeta\rangle \propto$ $\left(n_{c h} l^{3} N\right)^{-3 / 4}$. This is again a well confirmed result $[83,84]$ for the correlation length in semidilute polymer solutions. In the case of diluted solutions Eqn. (48) is also correct.

Finally, let us notice that $1 / z$ is the fractal dimension of the polymer chain and if we rewrite Eqn. (48) as

$P_{\text {void }}(\zeta, N) \propto \exp \left[-n_{\text {fract }} \zeta^{d}-n_{\text {fract }} N l^{d_{f}} \zeta^{\left.d-D_{f}\right]}\right.$

it will describe the distribution of voids in $d$-dimensional space with randomly placed, with mean concentration $n_{\text {fract }}$, fractals of mass $N$ and dimensionality $D_{f}$.

(b) Many-chain effects in trapping reactions

We are now in a position to extend the analysis of many-trap effects, presented in Section 2, to the examination of trapping kinetics in polymer systems (Fig. 2). Following [66-68] we represent the concentration of particles A at time $t$ as

$$
\frac{C(t)}{C(0)} \propto\left\langle P_{\text {void }}(\zeta, N) P(\zeta, t)\right\rangle
$$

where the average is taken over all cavities; $P_{\text {void }}(\zeta, N)$ is defined by our Eqn. (48) and $P(\zeta, t)$ (Eqn. (25)) is the probability that a particle located in the spherical cavity of radius $\zeta$ until time $t$ does not reach its boundary. Therefore, one has to analyze the behavior of the following integral

$$
\frac{C(t)}{C(0)} \propto \int_{0}^{\infty} d L \zeta^{d-1} \exp \left[-n_{c h} \zeta^{d}-n_{c h} N l^{1 / z} \zeta^{d-1 / z}-D t / \zeta^{2}\right]
$$

This analysis can also be performed using the steepest descent method. First of all let us consider the limit of very large times, $D t>>$ 
$n_{c h} N^{z(d+2)}$. Within this limit the second term in the exponent is small compared with the first one and the integral in Eqn. (49) casts into the form (27). Thus one arrives at the universal long-time decay which stems from the large-scale properties of polymer solutions (is independent of the chain's conformations and thus of the chain length $N$ ) and is quite similar to the decay found for the system of uncorrelated point traps

$\log \left(\frac{C(t)}{C(0)}\right) \propto-n_{c h}^{2 / d+2}(D t)^{d / d+2}$

The important difference is, however, that the factor in the exponent is proportional to the power of the concentration of polymers, but not to the actual concentration of traps $n_{c h} N$. In this regime each polymer acts effectively as a single trap.

Consider next the intermediate time behavior. At intermediate times, conversely, the second term in the exponent dominates and one obtains a conformation-dependent decay law of the form

$\log \left(\frac{C(t)}{C(0)}\right) \propto-\left(n_{c h} N\right)^{2 z /(z d+2 z-1)}(D t)^{(z d-1) /(z d+2 z-1)}$

For example, in the case of $3 \mathrm{~d}$ solution of Gaussian polymers Eqn. (51) predicts

$\log \left(\frac{C(t)}{C(0)}\right) \propto-\left(n_{c h} N\right)^{2 / 3}(D t)^{1 / 3}$

and for $3 \mathrm{~d}$ solutions of swollen coils

$\log \left(\frac{C(t)}{C(0)}\right) \propto-\left(n_{c h} N\right)^{3 / 5}(D t)^{2 / 5}$

These decay laws exhibit substantially slower behavior than the universal long-time dependence in Eqns. $(27,50)$.

Let us present some estimates of the crossover times and reaction depths. Consider the case of $3 d$ concentrated polymer solutions, i.e. Gaussian chains presented at high concentration, so that the volume fraction of polymers $\phi$ is near unity, $\phi \propto 1$. Summing up the results presented in Sections 3 and 4 one can expect the following kinetic scenario. At small times the decay is governed by the transient mean-field 
dependence in Eqn. (44), associated with the formation of the depletion zone around each chain. We will call this regime A. At greater times, i.e. times at which the Smoluchowski-like constant approaches a steadystate value in Eqn. (43) the decay of particles concentration will be purely exponential (regime B). At greater times one can expect the appearance of conformation-dependent stretched-exponential dependence in Eqn. (52) associated with many-chain effects (regime C), which, in the limit of very large times will be followed by the universal dependence in Eqn. (50) (regime D). However, it turns out that in the concentrated polymer solutions this scenario does not work. First we estimate the time at which the steady state mean-field regime $\mathrm{B}$ is established. This time grows linearly with $N, D t_{A \rightarrow B} \propto N$. Next, comparing the exponents in the steady state mean-field dependence (B) and the intermediate time fluctuationinduced behavior (C) we find that $t_{B \rightarrow C} \propto N^{3 / 4} \phi^{-1 / 2}$. Therefore, for concentrated solutions of Gaussian polymers the steady state mean-field dependence (B) will not exist, since $t_{B \rightarrow C} \ll<t_{A \rightarrow B}$. The crossover time $t_{A \rightarrow C}$ $\propto \phi^{-2}$ is also small in the concentrated solutions and, therefore, the transient mean-field dependence A can hardly be observed. Thus, in the concentrated solutions fluctuation-induced laws will describe the kinetics of trapping reactions over the entire time domain. The crossover time from the conformation-dependent law in Eqn. (52) to the universal decay in Eqn. (50) is of the order of $t_{C \rightarrow D} \propto \phi N^{3 / 2}$, i.e. can be very large if $N$ is large. Besides, the correspondent reaction depth is exponentially small, $C\left(t_{C \rightarrow D}\right) / C(0) \propto \exp (-\phi \sqrt{N})$, i.e. one can infer that the bulk of particles $A$ will be trapped via the conformation-dependent law in Eqn. (52). The numerical simulation results [100] confirm the fluctuation-induced decay forms and show that they are valid beginning from the earliest stages of reaction. In the case of diluted polymer solutions the volume fraction of polymers appears as an additional controlling parameter and the succession of regimes $A \rightarrow B \rightarrow C \rightarrow D$ can be, in principle, observed depending on the relation between $N$ and $\phi$.

\subsection{Influence of fluctuations on the kinetics of recombination reactions in polymer systems}

Here we will present simple estimates of the magnitude of fluctuation effects in the kinetics of recombination reactions (IIa) involving reactive particles attached to polymers. Let us note that arguments invoked for the derivation of the estimate in Eqn. (21) are still valid and the behavior of particle concentrations is dominated by fluctuations of the difference between numbers of $\mathrm{A}$ and $\mathrm{B}$ particles in certain domains. However, 
"compact exploration" motion of particles drastically changes the forms of fluctuation-induced decay in Eqn. (21). The point is that the growth of the domain's size with time is completely different from that in systems with conventional diffusion of reactive species. In the case of non-diffusive random motion of particles the intermediate-time growth of $\zeta$ will be guided by Eqn. (28), i.e. $\zeta \propto t^{v / 2}$ with $v=1 / 2$ for Rouse polymers and $v=$ $1 / 4$ for reptation model $*$. At times greater than $t_{\text {char }} \propto N^{\beta}$ with $\beta=2$ for the Rouse model and $\beta=3$ for reptation model the diffusive growth is restored, $\zeta \propto \sqrt{N^{1-\beta} t}$. Therefore, the modified due to anomalous transport properties of reactants fluctuation-induced behavior in Eqn. (21) will be characterized by the following dependences. At times less than $t_{\text {char }}$

$C(t) \propto \sqrt{C(0)} t^{-d v / 4}$

and at greater times $\left(t \geq t_{\text {char }}\right)$

$C(t) \propto \sqrt{C(0)} N^{(\beta-1) / 4} t^{-d / 4}$

Next, let us estimate the crossover times between the mean-field kinetics predicted in [31] and fluctuation-induced asymptotic forms in Eqns. $(54,55)$. One can expect the following succession of dynamical regimes in $3 \mathrm{~d}$ systems. The first stage will be governed by the mean-field "compact exploration" regime $(\mathrm{A}), C(t) \propto C(0) t^{-3 v / 2}$, Eqn. (31). At greater times this dependence will be followed by mean-field dependence, $C(t) \propto$ $N^{\beta-1} t^{-1}$, associated with the steady state Smoluchowski constant (regime B). Eventually, one expects the appearance of the fluctuation-induced dependence in Eqn. (54) (regime C) which will be followed by the longtime asymptotic form in Eqn. (55) (regime D). The crossover time from A to $\mathrm{B}$ is simply $t_{\text {char }}$ which grows with $N$ as $N^{\beta}$. Comparing A and Eqn. (54) we find that this decay becomes comparable at much less time $t_{A \rightarrow C} \propto$ $\varepsilon^{2 / 3 v}$, where $\varepsilon$ is the initial volume fraction of reactants. Therefore, we infer that stage B simply does not exist. Besides, time $t_{A \rightarrow C}$ is very small and regime A can hardly be observed as an intermediate asymptotic form. Thus, we can conclude that in the systems under consideration fluctuation-induced laws in Eqns. $(54,55)$ will describe reaction kinetics over the entire time domain.

* Of course, the decay of correlation functions is also affected by "compact exploration" motion of active particles. At the intermediate times $P_{A B}(t)$ decays as $P_{A B}(t) \propto-1 / \zeta^{d}$. 


\section{CONCLUSIONS}

To summarize, we have considered different types of chemical bimolecular reactions involving polymer chains. These are binary and recombination reactions with particles of reactants attached to movable polymers in polymer solutions and trapping reaction in systems with polymerized traps. We have discussed both mean-field considerations, based on the Smoluchowski-de Gennes approach, and many-particle treatments associated with spatial fluctuations in particle concentrations.

The guideline of this paper is the idea of "compact" and "non-compact exploration" types of random motion proposed by de Gennes in his paper [31] on the kinetics of chemical reactions involving polymers. Here we have based most of our analysis on this approach. We have shown that it is fruitful not only for the kinetic description of mean-field behavior, but also for the estimates of fluctuation effects and fluctuation-induced kinetics and provides a clear picture of the underlying physics.

We have illustrated, in terms of mean-field considerations, that the presence of polymers essentially influences reaction kinetics, ultimately resulting in non-traditional anomalous behavior as compared to the systems of monomeric reactants executing conventional diffusive motion. The origin of this distinction is caused by specific transport properties of polymers ("compact exploration") and essential correlations which polymers introduce into the reactive systems. Besides, we have shown that the presence of polymers drastically enhances fluctuation effects. In contrast to the systems of monomeric particles in which such type of effects influences only the long-time asymptotic behavior, in polymer systems many-particle effects become decisive from essentially earlier times and dominate the conversion of the bulk of reactive species.

\section{ACKNOWLEDGEMENTS}

The authors have greatly benefited from helpful discussions with J.C. Andre, F. Baros, A. Blumen, E. Clement, B. Gaveau, A. Perera and J.M. Deutch. G.O. gratefully acknowledges the fellowship by the French Ministry of Research and Technologies.

\section{REFERENCES}

1 S.A. Rice, Diffusion-Limited Reactions, in: C.H. Bamford, C.F.H. Tipper and R.G. Compton (Eds.), Chemical Kinetics, Vol. 25. Elsevier, Amsterdam, 1985.

2 C.W. Gardiner, Handbook of Stochastic Methods, Springer, Berlin, 1983. 
3 Chemical Reactivity in Liquids, eds. M. Moreau and P. Turq, Plenum, New York, 1988.

4 T.R. Waite, Phys. Rev., 107 (1957) 463.

5 G.H. Weiss, J. Stat. Phys., 42 (1986) 3.

6 D. Calef and J.M. Deutch, Annu. Rev. Phys. Chem., 34 (1983) 493.

7 P. Hanggi, P. Talkner and M. Borkovec, Rev. Mod. Phys., 62 (1990) 251.

8 S.F. Burlatsky and G.S. Oshanin, J. Stat. Phys., 65 (1991) 1095.

9 G.S. Oshanin and S.F. Burlatsky, J. Stat. Phys., 65 (1991) 1109.

10 S.F. Burlatsky, G.S. Oshanin and A.A. Ovchinnikov, in: Low Dimensional Conductors and Superconductors, Eds., A.A. Ovchinnikov and I.I. Ukrainskii, Springer Research Reports in Physics, Springer, Berlin, 1991, p.129.

11 A. Blumen, Nuovo Cimento B, 63 (1981) 50.

12 A. Blumen, J. Klafter and G. Zumofen, in: I. Zschokke (Ed.), Optical Spectroscopy of Glasses. Reidel Publ. Company, 1986, p.199.

M. von Smoluchowski, Z. Phys. Chem., 92 (1917) 129. P. Debye, Trans. Electrochem. Soc., 82 (1942) 265.

15 F.C. Collins and G.E. Kimball, J. Colloid. Sci., 4 (1949) 425.

16 L. Monchik, J.L. Magee and A.H. Samuel, J. Chem. Phys., 26 (1957) 935.

17 J.M. Deutch and B.U. Felderhof, J. Chem. Phys., 59 (1973) 1669.

18 P.G. Wolynes and J.M. Deutch, J. Chem. Phys., 65 (1976) 450.

19 B. Gaveau, J.T. Hynes, R. Kapral, and M. Moreau, J. Stat. Phys., 56 (1989) 879; 895; D. Borgis and M. Moreau, Physica A, 163 (1990) 877.

20 J.C. Andre, F. Baros, M. Bouchy and J.P. Badiali, Chem. Phys., 103 (1986) 391.

21 G. Oshanin, M. Mogutov, M. Moreau, and S.F. Burlatsky, to appear in the J. of Molecular Liquids.

22 P. Argirakis and R. Kopelman, J. Chem. Phys., 72 (1980) 3053; Chem. Phys. Lett., 61 (1979) 187.

23 S.F. Burlatsky, O.F. Ivanov and J.M. Deutch, J. Chem. Phys., 97 (1992) 156.

H. Schnorer, I.M. Sokolov, and A. Blumen, Phys. Rev. A, 42 (1990) 7075; 44 (1991) 2388.

25 D. Borgis, B. Gaveau and M. Moreau, J. Stat. Phys., 45 (1986) 319.

26 D. Borgis and M. Moreau, J. Stat. Phys., 37 (1984) 6131.

27 S. Lee and M. Karplus, J. Chem. Phys., 86 (1987) 1883.

28 A. Szabo, J. Chem. Phys., 95 (1991) 2481.

29 A. Perico and C. Cuniberti, J. Polymer Sci. Polymer Phys., Ed. 15 (1977) 1435.

30 B. Friedman and B. O'Shaughnessy, Europhys. Lett., 21 (1993) 779.

31 P.G. de Gennes, J. Chem. Phys., 76 (1982) 3316; 3322.

32 G. Wilemski and M. Fixman, J. Chem. Phys., 58 (1973) 5009; 60 (1974) 866; 878.

33 M. Doi, Chem. Phys., 9 (1975) 455; 11 (1975) 107, 115.

34 B. Friedman and B. O'Shaughnessy, Phys. Rev. Lett., 60 (1988) 64; Phys. Rev. A, 40 (1989) 5950.

35 B. Friedman and B. O'Shaughnessy, J. Phys., II, 1 (1991) 471.

36 J. Hoshen and R. Kopelman, J. Chem. Phys., 65 (1976) 2817.

37 G. Zumofen and A. Blumen, Chem. Phys. Lett., 88 (1982) 63; A. Blumen, G. Zumofen and J. Klafter, Phys. Rev. B, 30 (1984) 5379.

38 H.B. Rosenstock, SIAM J. Appl. Math., 9 (1961) 169; Phys. Rev. A, 187 (1969) 1166.

39 A. Dvoretzky and P. Erdos, in: Proc. Second Berkeley Symposium (1950) 353.

40 S.F. Burlatsky and A.A. Ovchinnikov, Sov. Phys. JETP, 65 (1987) 908.

41 T. Eisele and R. Lang, Prob. Th. Rel. Fields, 74 (1987) 125. 
A.M. Berezhkovski, Yu.A. Makhnovski and R.A. Suris, J. Stat. Phys., 57 (1989) 333; J. Stat. Phys., 65 (1991) 1025.

L. Onsager, Phys. Rev., 54 (1938) 554.

K.M. Hong and J. Noolandi, J. Chem. Phys., 68 (1978) 5163; 5172.

R. Kopelman, Science, 241 (1988) 1620.

E. Clement, L.M. Sander and R. Kopelman, Phys. Rev. A, 39 (1989) 6472; A 40 (1989) 6455; 40 (1989) 6466.

I.M. Sokolov, Phys. Lett. A, 139 (1989) 403.

G.S. Oshanin, S.F. Burlatsky and A.A. Ovchinnikov, Phys. Lett. A, 139 (1989) 245. S.F. Burlatsky, P.P. Levine, I.V.
Chem. Phys. Lett., 66 (1979) 565.

N. Agmon, J. Chem. Phys., 81 (1984) 2811.

S.H. Northrup and J.T. Hynes, J. Stat. Phys., 18 (1978) 91.

J. Duhamel, M.A. Winnik, F. Baros, J.C. Andre and J.M. Martinho, J. Phys. Chem., 96 (1992) 9805.

J.C. Andre, F. Baros and M.A. Winnik, J. Phys. Chem., 94 (1990) 2942.

A.A. Ovchinnikov and Ya.B. Zeldovich, Chem. Phys., 28 (1978) 215.

S.F. Burlatsky, Teor. Exp. Chem., 14 (1978) 343.

D. Toussaint and F. Wilczek, J. Chem. Phys., 78 (1983) 2642.

P. Meakin and H.E. Stanley, J. Phys. A, 17 (1984) L173.

G. Zumofen, A. Blumen and J. Klafter, J. Chem. Phys., 82 (1985) 3198.

K. Kang and S. Redner, Phys. Rev. Lett., 52 (1984) 955.

(1)
(1991) 297.

S.F. Burlatsky, G.S. Oshanin and A.A. Ovchinnikov, Sov. Phys. JETP, 68 (1989) 977. S.F. Burlatsky, G.S. Oshanin and A.A. Ovchinnikov, Chem. Phys., 152 (1991) 13.

G.S. Oshanin, A.I. Chernoutsan and S.F. Burlatsky, Theor. Exp. Chem. 12 (1990) 1. G.S. Oshanin, A.A. Ovchinnikov and S.F. Burlatsky, J. Phys. A, 22 (1989) L977.

G.S. Oshanin and S.F. Burlatsky, J. Phys. A, 22 (1989) L973.

G.S. Oshanin, Sov. J. Chem. Phys., 9 (1990) 246.

B.Ya. Balagurov and V.G. Vaks, Sov. Phys. JETP, 38 (1974) 968.

M.D. Donsker and S.R.S. Varadhan, Comm. Pure Appl. Math., 28 (1975) 525.

P. Grassberger and I. Procaccia, J. Chem. Phys., 77 (1982) 628.

R.F. Kayser and J.B. Hubbard, Phys. Rev. Lett., 51 (1982) 6281.

T.C. Lubensky, Phys. Rev. A, 30 (1984) 2657.

S.R. Renn, Nucl. Phys. B, 275 (1986) 273.

R.A. Auerbach and G.L. McPherson, Phys. Rev. B, 33 (1986) 6815.

U. Seiferheld, H. Bassler and B. Movaghar, Phys. Rev. Lett., 51 (1983) 813.

I.G. Hunt, D. Bloor, and B. Movaghar, J. Phys. C, 18 (1985) 3497.

Ya.B. Zeldovich and A.A. Ovchinnikov, Sov. Phys. JETP, 47 (1978) 82; JETP Lett., 26 (1977) 440.

N. Agmon and A. Szabo, J. Chem. Phys., 92 (1990) 5270.

W. Naumann, J. Chem. Phys., 98 (1993) 2353.

S.F. Burlatsky, Sov. Phys. Doklady, 286 (1986) 155.

P.E. Rouse, J. Chem. Phys., 21 (1953) 1272.

P.G. de Gennes, Scaling Concepts in Polymer Physics (Cornell University, Ithaca, 1979). 
84 M. Doi and S.F. Edwards, The Theory of Polymer Dynamics, Clarendon Press, Oxford 1986.

85 M. Doi and S.F. Edwards, J. Chem. Soc. Faraday Trans. 2, 74 (1979) 1789; 1802; 1818.

86 T.P. Lodge, N.A. Rotstein and S. Prager, Adv. Chem. Phys., 79 (1990).

87 I. Szleifer and R.F. Loring, J. Chem. Phys., 95 (1991) 2080.

88 A. Baumgärtner, in Polymer Motion in Dense Systems, Eds.: D. Richter and T. Springer, Springer, Berlin 1987.

89 P.T. Callaghan and A. Coy, Phys. Rev. Lett., 68 (1992) 3176.

90 J. Machta, Phys. Rev. A, 40 (1989) 1720; see also: M. Sahimi and V.L. Jue, Am. Inst. Chem. Eng. Symp. Ser., 84 (1988) 40.

91 V.S. Nanda and S.C. Jain, J. Chem. Phys., 49 (1968) 1318.

92 A.M. Gupta, R.C. Hendrickson and C.W. Macosko, J. Chem. Phys., 95 (1991) 2097.

93 U.S. Agarwal and D.V. Khakhar, J. Chem. Phys., 96 (1992) 7125.

94 G. Oshanin and M. Moreau, to be published.

95 P.G.J. van Dongen and M.H. Ernst, Phys. Rev. Lett., 54 (1985) 1396; P. Meakin and M.H. Ernst, Phys. Rev. Lett., 60 (1988) 2503; F. Family, P. Meakin and D.M. Deutch, Phys. Rev. Lett., 57 (1987) 727; P.G.J. van Dongen, Phys. Rev. Lett., 63 (1989) 1281.

96 P. Meakin, Physica A, 165 (1990) 1; R. Jullien, New J. Chem., 14 (1990) 239.

97 S.F. Burlatsky, G.S. Oshanin and V.N. Likhachev, Sov. J. of Chem. Phys., 7 (1988) 970.

98 M.E. Cates and T.A. Witten, Phys. Rev. A, 35 (1987) 1809.

99 S.F. Burlatsky and G.S. Oshanin, Phys. Lett., 145 (1990) 61.

100 G.S. Oshanin, A. Mogutov and S.F. Burlatsky, Phys. Lett. A, 149 (1990) 55.

101 T. Förster, Z. Naturforsch. Teil A, 4 (1949) 321.

102 M. Inokuti and F. Hirayama, J. Chem. Phys., 43 (1965) 1978.

103 A.I. Burshtein, Sov. Phys. Usp., 27 (1984) 579.

104 J. Klafter and A. Blumen, J. Chem. Phys., 80 (1984) 875; A. Blumen, J. Klafter and G. Zumofen, J. Chem. Phys., 84 (1986) 1397.

105 G.H. Frederickson, H.C. Andersen and C.W. Frank, Macromolecules 16 (1983) 1456; J. Chem. Phys., 79 (1983) 3572.

106 S.F. Burlatsky, G.S. Oshanin and A.A. Ovchinnikov, Phys. Lett. A, 139 (1989) 241.

107 U. Even, K. Rademann, J. Jortner, N. Manor and R. Reisfeld, Phys. Rev. Lett., 52 (1984) 2164.

108 A. Grinvald, E. Haas and I.Z. Steinberg, Proc. Natl. Acad. Sci. USA, 69 (1972) 2273.

109 E. Haas, M. Wilchek, E. Katchalskii-Katzir and I.Z. Steinberg, ibid., 72 (1975) 1807.

110 O. Pekcan, M.A. Winnik and M.D. Croucher, Phys. Rev. Lett., 61 (1988) 641.

111 A.K. Roy and A. Blumen, J. Chem. Phys., 91 (1989) 4353.

112 S.F. Burlatsky, G.S. Oshanin and A. Mogutov, Phys. Rev. Lett., 65 (1990) 3205.

113 A. Blumen and J. Manz, J. Chem. Phys., 71 (1979) 4694.

114 K. Allinger and A. Blumen, J. Chem. Phys., 72 (1980) 4608.

115 G. Oshanin, O.F. Ivanov and S.F. Burlatsky, to be published. 